\title{
Improvement of Three Dimensional Acoustic Field Estimation Using Tomographic Reconstructions of the Ocean
}

\author{
by \\ Elizabeth Anne Rowe \\ B.S., United States Naval Academy (1980) \\ Submitted in partial fulfillment of the \\ requirements for the degree of \\ MASTER OF SCIENCE IN OCEAN ENGINEERING \\ at the \\ MASSACHUSETTS INSTITUTE OF TECHNOLOGY \\ and the \\ WOODS HOLE OCEANOGRAPHIC INSTITUTION
}

August 1988

(C) Elizabeth Anne Rowe, 1988

The author hereby grants to MIT and WHOI permission to reproduce and to distribute copies of this thesis document in whole or in part.

Signature of Author

Joint Program in Oceanographic Engineering Massachusetts Institute of Technology

Woods Hole Oceanographic Institution August 5, 1988

Certified by

Dr. James F. Lynch Woods Hole Oceanographic Institution Thesis Supervisor

Certified by

Dr. Arthur B. Baggeroer Massachusetts Institute of Technology

Accepted by Dr. W. Kendall Melville Chairman,Joint Committee for Oceanographic Engineering Massachusetts Institute of Technology/Woods Hole Oceanographic Institution 


\title{
Improvement of Three Dimensional Acoustic Field Estimation Using Tomographic Reconstructions of the Ocean
}

by

\author{
Elizabeth Anne Rowe
}

\author{
Submitted to the Massachusetts Institute of Technology/ \\ Woods Hole Oceanographic Institution \\ Joint Program in Oceanographic Engineering \\ on August 5, 1988, in partial fulfillment of the \\ requirements for the degree of \\ Master of Science in Ocean Engineering
}

\begin{abstract}
In order to determine the efficacy of tomographic reconstructions of the ocean sound speed structure in improving acoustic field predictions for source localization, a $150 \mathrm{~km}$ by 350 $\mathrm{km}$ volume of ocean 3000 meters deep was synthetically modeled to be similar to the Gulf Stream system, including an eddy and a front. The features were Gaussian, with the eddy's maximum sound speed perturbation being $10 \mathrm{~ms}^{-1}$ and the front's maximum perturbation $15 \mathrm{~ms}^{-1}$. Two vertical slices through this system were inverted in a synthetic tomography experiment using linear optimal estimation theory. Inversions were also performed using $\mathrm{XSV}$ and satellite sea surface temperature data. Gaussian fits to the reconstructed features were constructed for use with a three dimensional raytrace program (HARPO). Three dimensional rays were propagated both through the reconstructions and the original model. Travel time versus intensity (transmission loss) for the eigenrays was used as a basis for intercomparison. Tomographic results showed good reconstruction for a first iteration of the inversion, but inadequate vertical resolution. Iterations and the use of more refractive eigenrays are needed for improvement of the reconstruction, especially for the front. Reconstructed results for the acoustic field should improve conventional beamforming, but are probably inadequate for matched field processing.
\end{abstract}

Thesis Supervisor: Dr. James F. Lynch

Woods Hole Oceanographic Institution 


\section{Acknowledgements}

For invaluable guidance and encouragement, I would like to thank Professor Jim Lynch, without whom I could not have reached my goal. In addition, Padmaraj Vengayil, Arthur Newhall, Wendy Lawrence, John Daugherty, Chi-Fang Chen, Stanley Rosenblad, Markku Santala, Mike Byman, Andy Trivett and Maxine Jones provided the friendship and assistance I needed to get through each of the steps required to complete this program. I would like to thank the United States Navy, the Massachusetts Institute of Technology and the Woods Hole Oceanographic Institution for giving me this extraordinary opportunity. I would also like to thank Susan L. K. Briggs for her support, advice and friendship. To my mother and father, who listened across the miles to each difficulty I encountered, I give my heartfelt thanks. To my daughter Rebecca, I give my love for her smiles and laughter and even her tears which gave me such strength. To my husband Don, I give my deepest love and respect. His thoughtful advice and unending support got me through every obstacle. Finally, I thank God for all these things. 


\section{Contents}

1 Introduction $\quad 8$

1.1 objectives ...................... 8

1.2 Acoustic Tomography . . . . . . . . . . . . . . . 9

1.3 The Gulf Stream System . . . . . . . . . . . . . . . . 9

1.4 Ray Tracing ............................... 10

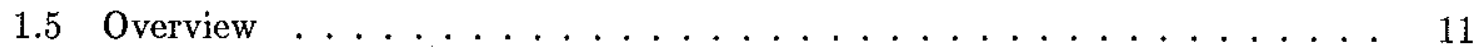

2 Components of the Analysis $\quad 12$

2.1 The General Problem ....................... 12

2.2 Two-Dimensional Raytracing . . . . . . . . . . . . . . 13

2.3 Three-Dimensional Raytracing . . . . . . . . . . . . . 14

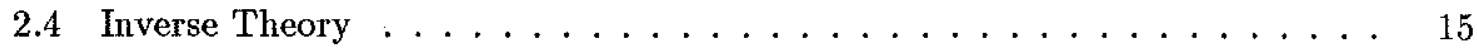

2.5 The Ocean Model . . . . . . . . . . . . . . . . . 16

2.6 Ocean Reconstruction and Propagation Modeling . . . . . . . . . . . 20

3 Results and Analysis $\quad 25$

3.1 Tomographic Reconstructions . . . . . . . . . . . . . . 25

3.2 Three Dimensional Propagation . . . . . . . . . . . . . . . 36

4 Conclusions $\quad 49$ 


\section{List of Figures}

$2-1$ Gulf Stream sound speed profile . . . . . . . . . . . . . 18

2-2 Top view of the ocean model geometry, including source and receiver positions 19

2-3 Vertical slice of input model for eddy and front, from $S 1$ to $R 2 \ldots \ldots 21$

2-4 Vertical slice of input model for eddy and front, from $S 2$ to $R 1 \ldots 22$

2-5 Diagram for calculation of transmission loss due to geometrical spreading . 24

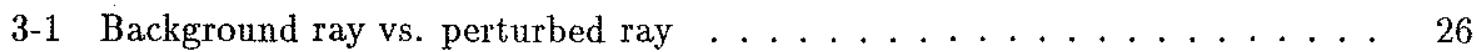

3-2 Sound speed perturbation estimate $\left(\mathrm{ms}^{-1}\right)$ using "pure acoustics" . . . . 28

3-3 Horizontal and vertical resolution lengths for "pure acoustics" . . . . . . 29

3-4 Sound speed perturbation estimate $\left(\mathrm{ms}^{-1}\right)$ using acoustics and satellite sea surface temperature measurements $\ldots \ldots \ldots \ldots$

3-5 Horizontal and vertical resolution lengths for acoustics and satellite data . 31

3-6 Sound speed perturbation estimate $\left(m s^{-1}\right)$ using acoustics and XSV data . 32

3-7 Horizontal and vertical resolution lengths for acoustics and XSV data . . . 33

3-8 Sound speed perturbation estimate $\left(\mathrm{ms}^{-1}\right)$ using acoustics, satellite and XSV

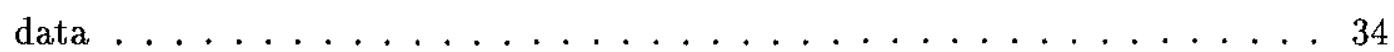

3-9 Horizontal and vertical resolution lengths for acoustics, satellite and XSV

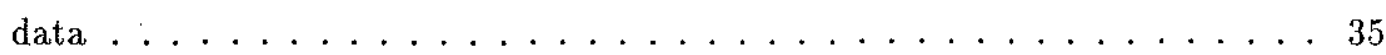

3-10 Sound speed perturbation estimate $\left(m s^{-1}\right)$ using XSV and satellite data, no

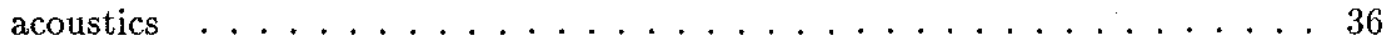

3-11 Horizontal and vertical resolution lengths for XSV and satellite data, no

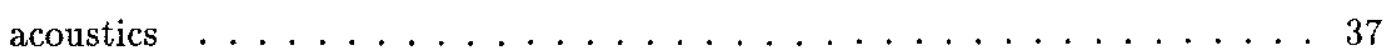

$3-12$ Gaussian fit to inversion with acoustics, path $S 1$ to $R 2 \ldots \ldots \ldots$ 
$3-13$ Gaussian fit to inversion without acoustics, path $S 1$ to $R 2 \ldots \ldots$

3-14 Model vs. background raypaths . . . . . . . . . . . . . 42

3-15 Model vs. inverse with acoustics raypaths . . . . . . . . . . . 43

3-16 Model vs. inverse without acoustics raypaths . . . . . . . . . . . . . 44

3-17 Two rays through the model, horizontal and vertical aspect . . . . . . . . 45

3-18 Transmission loss versus time of ray arrivals for the background profile and the model . . . . . . . . . . . . . . . 47

3-19 Transmission loss versus time of ray arrivals for the inversion with acoustics and the inversion without acoustics $\ldots \ldots \ldots \ldots$ 


\section{List of Tables}

3.1 CBLOB3 (equation 2.10) data for model, inversion with acoustics, and in-

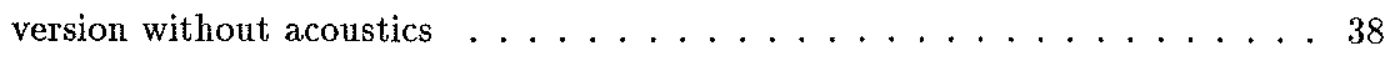




\section{Chapter 1}

\section{Introduction}

\section{$1.1 \quad$ Objectives}

Ocean acoustic tomography (Munk and Wunsch, 1979) is a useful techinique for reconstructing mesoscale ocean features. This thesis will address the following issues in ocean acoustic tomography:

- The efficacy of tomography, compared to conventional methods, in reconstructing the sound speed structure of a three dimensional ocean volume.

- The information, other than "pure acoustics", needed for effective inversion when strong sound speed perturbations are encountered.

- The stability of rays which propagate through strong sound speed perturbations.

- The differences between three dimensional rays propagating through a real ocean and those propagating through a reconstructed model of the ocean.

Addressing these issues will help clarify the importance of acoustic tomography in reconstructing sound speed perturbations due to the ocean mesoscale, and particularly its effect upon improving three dimensional propagation estimates which are needed for signal processing and source localization. To address these issues, we will study the case of tomographically reconstructing a synthetic data Gulf Stream system, and seeing the effects on three dimensional propagation. 


\section{$1.2 \quad$ Acoustic Tomography}

A major problem in studying the ocean is the difficulty of obtaining good temporal and areal coverage by most observational methods. Data collection by shipborne sensors requires a great deal of time to observe an area. In addition, features can change during survey time resulting in poor resolution. Point sensors on moorings see only one point in the ocean over time. Aircraft flights have limited duration for data collection. Satellites cannot "see" the ocean interior because electromagnetic radiation cannot penetrate the water and, in addition, often have large time gaps between repeat orbits.

Ocean acoustic tomography is a possible solution to some of these problems. In doing tomography, sources and receivers (or transceivers) are placed in various positions in the ocean. Each source transmits a pseudorandom coded pulse (Eisler, 1982) which is detected and decoded at each receiver. The difference in travel-time for each path going through this volume compared to that computed for a background model is "inverted" to estimate sound speed anomalies. These anomalies can then be used to calculate ocean parameters such as temperature and (to a lesser extent) salinity, and thus can be used to help calculate the ocean dynamics of the area.

In tomography, if $S$ sources and $R$ receivers are placed around an ocean volume and $T$ multipaths can be identified between them, the result is $S \times R \times T$ pieces of data. Spot measurements, on the other hand, produce only $S+R$ pieces of data and these data are measured at only one point in the ocean at a time. Tomographic data gives a three dimensional measurement of ocean characteristics and the time required to collect this information is still relatively short. Acoustic tomography can potentially provide a real time three dimensional picture of ocean areas, if the ability to telemeter the data from remote moorings ever becomes available.

\subsection{The Gulf Stream System}

The time scale of interest for many mid-ocean eddies is approximately $50-150$ days (Spiesberger and Worcester, 1983). However, for the Gulf Stream, much shorter time scales are indicated. The Gulf Stream can meander by 20 kilometers in one day (Spiesberger, et 
$a l ., 1983)$ and its eddies move at a speed of $3-7$ kilometers per day (Richardson, 1976). The study of this western boundary current system, then, must be treated in a different light than mid-ocean eddies.

Temperature and salinity variations in the Gulf Stream system have a strong impact on ocean dynamics. Sound speed calculations in this area indicate characteristically large changes in these parameters. The front which separates colder slope water from the warm Sargasso Sea water has an average increase in sound speed of $30 \mathrm{~ms}^{-1}$ across it (Cornuelle and Malanotte-Rizzoli, 1986). It meanders through the western North Atlantic and sheds warm and cold "rings" (Fuglister, 1972). (This name was suggested by Fuglister because of the way these anomalies swirl and finally break off from the Gulf Stream, forming closed rings). The rings travel some distance, often rejoining the stream at a later time. They can be up to 200 kilometers wide and create very large sound speed changes. Cold rings are formed by cold slope water being entrained into the warmer Sargasso Sea and warm rings are formed in the opposite manner. These rings are the most energetic eddies in the ocean. They strongly affect the circulation, momentum and energy of the Gulf Stream system. Moorings cannot be put in the Gulf Stream easily because of strong currents. Measurements made by shipborne CTD's (Conductivity-Temperature-Depth probes) are highly accurate, but take a great deal of time. Therefore, rapid changes in temperature and salinity, characteristic of the Gulf Stream system, cannot be covered by these sensors on time scales equal to or smaller than the rate at which changes occur. The potential usefulness of acoustic tomography in this region is clear.

\subsection{Ray Tracing}

If an acoustic source produces sound with a wavelength which is small compared to the ocean feature length scales being examined, as it does in the work we will pursue, the geometrical optics approximation is valid. Ray theory can then be used to model the acoustic field. For deep water applications, such as the Gulf Stream system, ray theory requires far fewer terms than normal mode theory and provides a simpler physical picture of underwater sound transmission. Despite ray tracing's deficiencies in terms of caustics 
and diffraction, it is still a very good way to model sound propagation for range-dependent problems.

Until recently, only two-dimensional ray tracing programs have been used to describe ocean acoustic ray propagation, i.e. horizontal deflection of ray paths was not included. A true representation of the paths of rays through a three dimensional medium could not be accurately displayed. The changes in travel time due to horizontal deflections caused by very simple canonical eddy features were first estimated by Munk (1979), but more work using three-dimensional raytracing needed to be done. The three-dimensional raytracing program HARPO (Jones, et al., 1986a) was used to follow up Munk's work. It again used simple canonical features for demonstrating the three-dimensional effects. A method for finding eigenrays was developed to use with HARPO (Mercer, et al., 1985) for more general applications. This is the form of the program used for our work.

\subsection{Overview}

In Chapter 2, the ocean model used in this work will be described in detail. Following this, methods used for ray propagation calculations and the inversion of model travel time data are developed. A description of how inversion data are fit to a three dimensional canonical ocean model follows. Chapter 3 shows the results of the tomographic inversion using acoustics and then adding satellite sea surface temperature data and XSV data. Also, an inversion using the satellite and XSV data without acoustics will be performed. A twodimensional and finally three-dimensional Gaussian fit to the inversion which can be used with the three dimensional ray trace program will be shown. Three dimensional eigenrays will then be propagated through the background model, the true model and the three dimensional fits to the various inversions. Transmission losses and travel times will then be

calculated and compared between each of the cases. Chapter 4 contains a summary of the work and addresses future research in this area. 


\section{Chapter 2}

\section{Components of the Analysis}

\subsection{The General Problem}

In acoustic tomography, the travel time differences between pairs of eigenrays with the same history, one going through $a^{\prime}$ background profile and the other going through a perturbed profile or "real ocean", are the quantities which must be measured. These data are then used to find ocean sound speed perturbations, which are on the order of $m s^{-1}$, and currents, which are on the order of $\mathrm{cms}^{-1}$ (Munk and Wunsch, 1979). In our work, current effects will not be treated.

The travel time of any ray through a region is

$$
T_{i}=\int \frac{d s_{i}}{c(z, \theta, \phi)}
$$

where $d s_{i}$ is the differential arclength along the $i$ th raypath and $c$ is the local sound speed. It is usually assumed that the sound speed perturbations are linear about a background state, i.e.

$$
c(z, \theta, \phi)=c_{0}(z)+\delta c(z, \theta, \phi)
$$

where $c_{0}$ is the background state and $\delta c$ is the perturbation. Given that these sound speed perturbations are small, the travel time perturbation can be expressed as

$$
\delta T_{i}=\int \frac{\delta c(z, \theta, \phi)}{c_{0}^{2}(z)} d s_{i}
$$


where $d s_{i}$ is the unperturbed $i$ th raypath. Therefore, the perturbed and unperturbed paths must be close in trajectory to avoid error. For strong perturbations, this assumption may not hold and the problem becomes nonlinear. However, iterative techniques can often resolve these nonlinearities (Spofford and Stokes, 1984).

In order to find the travel times for the background profile, raytracing programs are standardly used. In our work, a model is used to generate the perturbed sound speed profiles and travel time data as well, i.e. we replace the field experiment part of the process with synthetic data.

\section{$2.2 \quad$ Two-Dimensional Raytracing}

Two dimensional ray tracing is a well known technique. It is computationally simpler than three dimensional ray tracing and is commonly used to approximate the ocean because out of plane effects are often small. Currently, all inverse techniques use such two dimensional codes, although a three dimensional inversion program is being developed. Two dimensional raytracing will be used in this work to construct inverse "slices" of the ocean, i.e. rays will be propagated along straight line paths (in the $x-y$ plane) through both the background and perturbed profiles. Travel time differences between eigenrays with identical ray histories will be measured and then inverted, resulting in an estimate of the sound speed perturbations along that path.

The raytrace program we used in our tomography inversions is MPP (Multiple Profile Program), written by C. W. Spofford. In MPP, the sound speed is linearly interpolated in range and depth in specified triangular regions. The sound-speed field is continuous everywhere, but the gradient of the sound-speed field is discontinuous at the triangular boundaries. The source and receiver depth must be specified, as well as the range of interest. Eigenrays are found by sending rays through this profile and then interpolating for exact elevation angles. The advantages of this program are that it is a fast code and produces ray histories in the appropriate format for the tomographic inverse. 


\subsection{Three-Dimensional Raytracing}

For three dimensional ocean features (fronts, eddies, etc.) or sloping bottoms, out of plane refraction can be an important feature of sound propagation. A three dimensional raytracing program is an effective means for looking at this effect. HARPO (Hamiltonian Acoustic Ray-Tracing Program for the Ocean) is a three dimensional program which was developed from earlier programs used for computing ray paths in the atmosphere (HARPA), and is the first effective three dimensional raytrace program available for general use.

An advantage of the HARPO code is that the models used to represent the ocean must have continuous gradients. Many models, like MPP, break the medium into linear segments or triangular cells within which ray paths are computed, which causes false caustics. HARPO avoids this problem. In addition, currents can be included in Hamiltonian raytracing.

In order to compute a raypath, the ocean must be modeled as a continuous three dimensional function with continuous gradients. Each raypath is calculated by numerically integrating Hamilton's equations with specified initial conditions. These include source location, direction of transmission (elevation and azimuth) and the surface and bottom models. The Hamiltonian is defined as

$$
H\left(x_{i}, k_{i}\right)=\left\{\omega-\vec{k} \cdot \vec{v}\left(x_{i}\right)\right\}^{2}-c^{2}\left(x_{i}\right) k^{2}
$$

where $x_{i}$ are coordinates of a point on the $i$ th raypath, $k_{i}$ are the wave number components, $\omega$ is the angular wave frequency, $v\left(x_{i}\right)$ is the ocean current, and $c\left(x_{i}\right)$ is the sound speed field. HARPO has many canonical models, in analytic form, which represent different ocean phenomena, including topography and mesoscale features, some of which we will use here. The program is also capable of utilizing user specified ocean feature models because of the separation of the models from the "background" ray computation.

The basic HARPO program does not accurately compute eigenrays. However, a means of doing this has been suggested (Mercer, et al., 1985) using HARPO ray history output. For this thesis this procedure was implemented for eigenray calculations. The method assumes that small changes in azimuth launch angle do not cause large changes in the azimuthal 
path of rays. This means that if one sends a ray directly toward the receiver and finds the deviation from this direction at the receiver range, changing the launch azimuth by that angle will cause the ray to approximately intersect the receiver. In addition, sending rays at small increments of elevation angle will result in finding two rays which bracket the receiver range at the receiver depth. Interpolating these rays will result in a ray which reaches the appropriate depth and range. The maximum deviation from receiver position allowed in our work is five meters.

\subsection{Inverse Theory}

Once the rays are traced through the background and perturbed profiles, the differences in travel times for rays with identical histories are computed as synthetic data for the inversion. The basic equation of inverse theory is

$$
G m+e=d
$$

where $d$ is the data vector (travel time perturbations), $e$ is the observational error (noise), $G$ is a known operator (the kernel), and $m$ is the model one is trying to estimate (sound speed perturbations). To solve this problem, linear optimal estimation theory (Liebelt, 1967) is used. In this method, $m$ is estimated in the form

$$
\hat{m}=A d
$$

where $\hat{m}$ is the estimate of the true model $m$. The solution lies in making the best choice of A. The linear inversion program used in our work, RSN2, was developed by C. S. Chiu (Chiu, et al., 1987) and solves the inverse problem by finding estimates which are linear combinations of the data with minimal mean square error (Liebelt, 1967). This error has two components; the variance, which is a measure of experimental noise, and the bias, which reflects the limited data available. This program takes a vertical ( $r-z$ plane) region which is to be inverted and breaks it into 225 rectangles of equal area. This is done by dividing both the ranges and depths into fifteen segments. The perturbations within each rectangle are assumed constant. It is assumed that random experimental noise is uncorrelated and has zero mean. The covariance of this noise is also assumed known. This is an underdetermined 
problem and therefore has an infinite number of possible solutions. However, requiring a solution which is linear with the data and results in minimum mean square error allows a single solution.

The Gauss-Markoff theorem states that the estimate of the model is

$$
\hat{m}=C_{\varepsilon} G^{T} C_{e}^{-1} d
$$

where

$$
C_{\varepsilon}=C_{m}^{-}-\left(C_{m} G^{T}\right)\left(G C_{m} G^{T}+C_{e}\right)^{-1}\left(C_{m} G^{T}\right)^{T}
$$

This is the covariance matrix of the total error, $\varepsilon=\hat{m}-m$. In equation 2.8 , the diagonal elements of $C_{\varepsilon}$ are the mean square errors of the estimates in each rectangle, $C_{e}$ is the covariance matrix of the noise $e$, and $C_{m}$ is the model covariance matrix. The anomalies are considered statistically homogeneous and the covariance function is assumed Gaussian in shape, i. e.

$$
C(\Delta r, \Delta z)=\sigma^{2} \exp -\left[\left(\Delta r / L_{r}\right)^{2}+\left(\Delta z / L_{z}\right)^{2}\right]
$$

where $\sigma^{2}$ is the variance of the perturbations, $\Delta r$ and $\Delta z$ are distances between points in a vertical slice, and $L_{r}$ and $L_{z}$ are the ocean correlation lengths. This type of function has been shown useful (Cornuelle, et al., 1985) for reconstructing mid-ocean eddies. The covariance matrix of the model, $C_{m}$, can be computed using this function. This a priori statistical information is sufficient to uniquely solve the inverse problem.

\subsection{The Ocean Model}

For our synthetic experiment, a section of ocean 350 kilometers by 150 kilometers was modeled. We wanted to create an environment similar to the Gulf Stream, but relatively simple, just including an eddy and a front. For ease of calculation the water depth was taken to be 3000 meters, less than that of the Gulf Stream region (approximately 5000 meters), and the bottom was assumed flat. A background sound-speed model, Figure 2-1, developed from historical data, was used (Cornuelle, 1982) for tracing background eigenrays. Sources were placed at 1050 meters depth and receivers at 1150 meters depth, just below the sound 
channel axis of the background profile. The depths for source and receiver were chosen to increase the number of eigenrays which can be resolved and identified (Cornuelle, 1983).

The three dimensional raytrace program HARPO has an ocean feature model available called CBLOB3, which can be used to create Gaussian sound speed perturbations decaying in all three spatial directions (Jones, et al., 1986a). The equation describing such anomalies is

$$
C(z, \theta, \phi)=C_{0}(z, \theta, \phi)\left\{1+\sum_{i=1}^{n} \nabla_{i} \exp \left[-\left(z-z_{i} / W_{z_{i}}\right)^{2}-\left(\theta-\theta_{i} / W_{\theta_{i}}\right)^{2}-\left(\phi-\phi_{i} / W_{\phi_{i}}\right)^{2}\right]\right\}
$$

where $C_{0}(z, \theta, \phi)$ is the background sound speed model, $\nabla_{i}$ is the fractional increase in sound speed of the $i$ th feature, $z_{i}$ is depth, $\lambda_{i}$ is latitude, $\theta_{i}=\pi / 2-\lambda_{i}, \phi_{i}$ is longitude, and $W_{z}, W_{\theta}$, and $W_{\phi}$ are the e-folding distances of the field in each of the three spatial directions. This three-dimensional perturbation model is used to model both the eddy and front.

The eddy chosen is a warm ring, simulating warm Sargasso Sea water entrained in the colder slope waters (Figure 2-2). A cold ring was not chosen because of their larger average size and greater sound speed anomalies (Robinson, 1983). The larger the sound speed anomalies are, the greater the nonlinearities, which would lessen the usefulness of our linear inversion technique. The eddy model chosen has a radius of 50 kilometers. A typical eddy, south of the Gulf Stream, has a $2-4^{\circ} \mathrm{C}$ temperature change from the surrounding ocean (Spiesberger,1983). In general

$$
\delta c / c=3.19 \times 10^{-3} \delta \theta
$$

where $\delta c$ is sound speed change in $m s^{-1}, c$ is background sound speed and $\delta \theta$ is temperature change in ${ }^{\circ} \mathrm{C}$. The 2 degree temperature change we chose for our calculations results in approximately $10 \mathrm{~ms}^{-1}$ maximum sound speed increase. This value falls to $10 \%\left(1 \mathrm{~ms}^{-1}\right)$ at 50 kilometers in latitude or longitude from the center. Its maximum value is at a depth of 400 meters and falls to $10 \%$ of that value at 1000 meters.

The front was the second Gaussian anomaly (Figure 2-2). However, it decays only in longitude and depth, not latitude. Although it is clear that the real Gulf Stream front shows an asymmetric increase in sound speed from the slope waters to the Sargasso Sea (Cornuelle and Malanotte-Rizzoli, 1986), a Gaussian model is chosen because of our restriction to the 


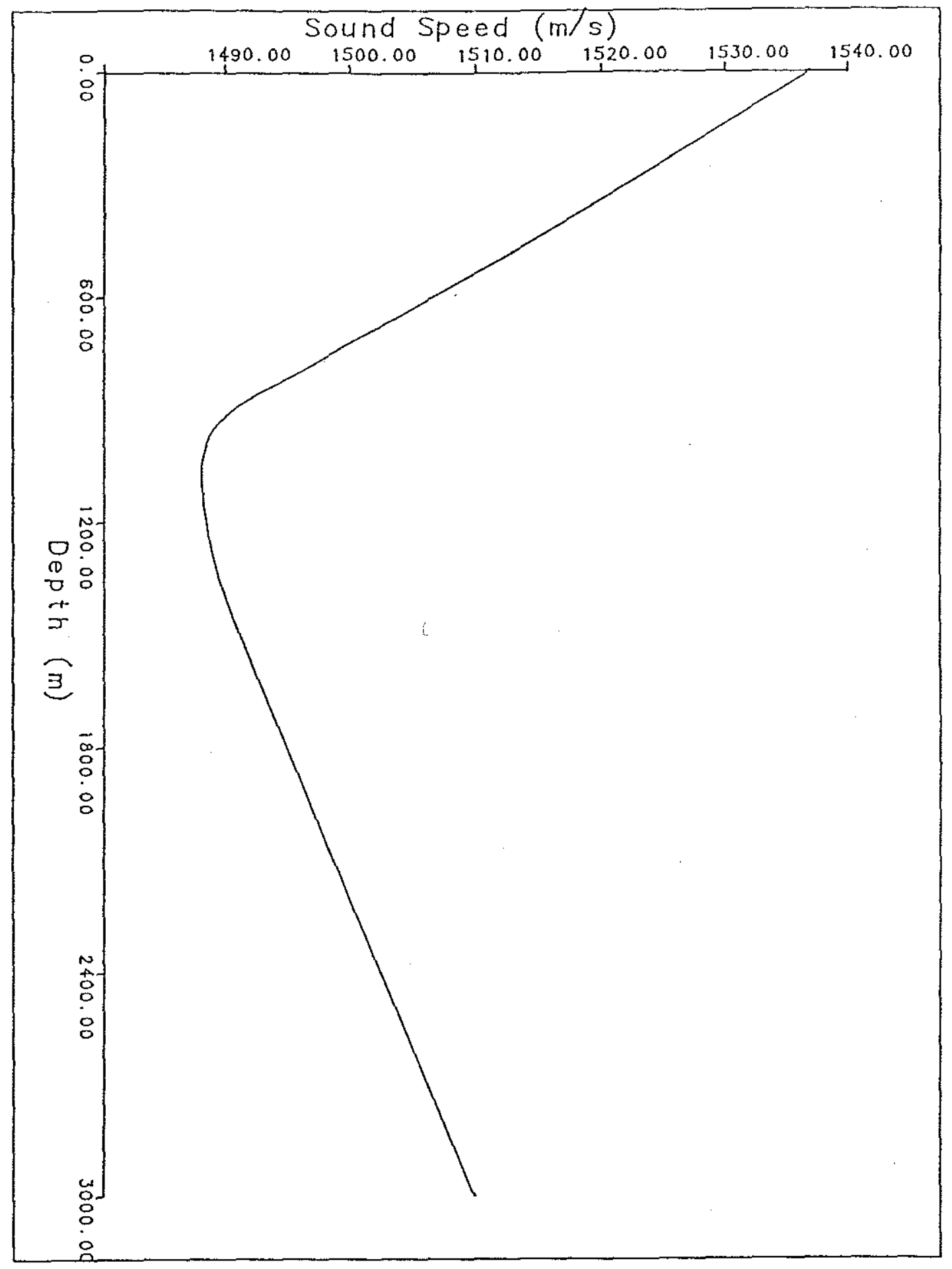

Figure 2-1: Gulf Stream sound speed profile 


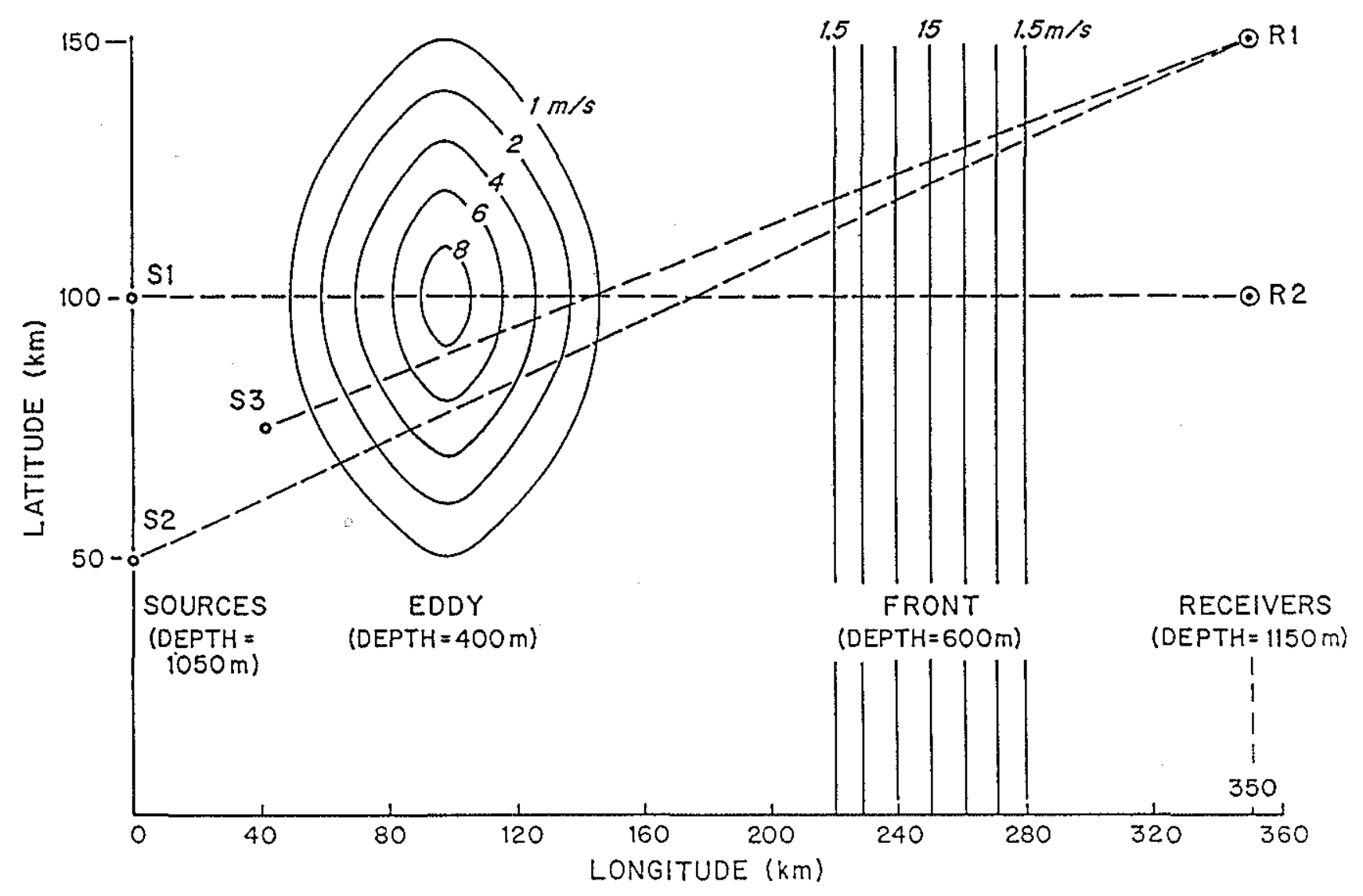

Figure 2-2: Top view of the ocean model geometry, including source and receiver positions 
use of CBLOB3. Also, the maximum sound speed change was chosen as $15 \mathrm{~ms}^{-1}$ despite the fact that it is often as much as $30 \mathrm{~ms}^{-1}$. Again, this was done to reduce the effects of large nonlinearities. The front decreases to $10 \%\left(1.5 \mathrm{~ms}^{-1}\right)$ at 30 kilometers longitudinally. Its maximum sound speed is at a depth of 600 meters and falls to $10 \%$ at 1500 meters. Both anomalies also show a surface expression. The surface sound speed values are simulated to be measured via satellite.

\subsection{Ocean Reconstruction and Propagation Modeling}

After creating this ocean model, sound speed perturbation data for individual slices between pairs of sources and receivers was needed for use in MPP, the two dimensional ray trace program. The first slice was between source $S 1$ and receiver $R 2$ (Figure 2-3). The sound speed anomalies for this slice were calculated by making $\theta$ constant in equation 2.10 . The second slice was between $S 2$ and $R 1$ (Figure 2-4). This data was calculated by using the angle from the horizontal along the path $\left(15.9^{\circ}\right)$ to find the latitude and longitude coordinates of the sound speed perturbations.

Rays were sent from source to receiver through these slices with elevation angles between $-20^{\circ}$ and $+20^{\circ}$, both through the background profile and the perturbed profile. Eigenrays which were both stable and identifiable were then matched between the background and the perturbed profiles. This was done by examining their ray histories and, at times, looking at the pictorial ray traces to see if their paths were approximately the same. These rays were then examined for differences in travel-time and this data was used for the inversion.

In addition to using "pure acoustics" (eigenrays only), XSV (expendable sound velocimeter) data and satellite sea surface temperature data were also used. This was because the "pure acoustics" inverse results were inadequate for these strong perturbations, as will be shown. In addition, these types of data can be collected in short periods of time and are therefore useful when "real time" reconstructions are desired. In order to better examine the benefits of acoustics, the inversions were also performed using only XSV and satellite data, without any travel time data. We then traced rays through these reconstructions.

Tomographic inverse results are not in canonical form, which made them difficult to use 


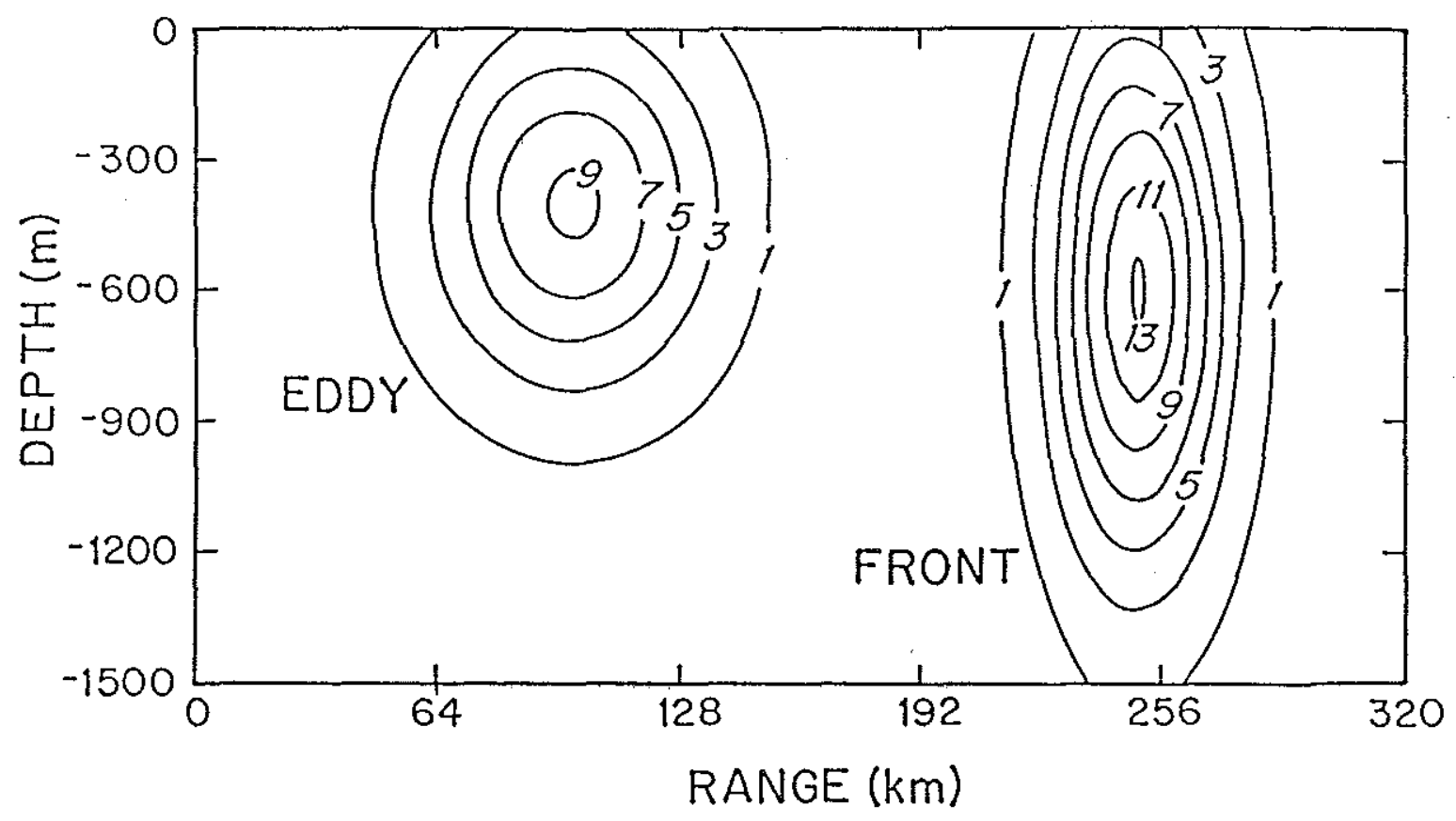

Figure 2-3: Vertical slice of input model for eddy and front, from $S 1$ to $R 2$ 


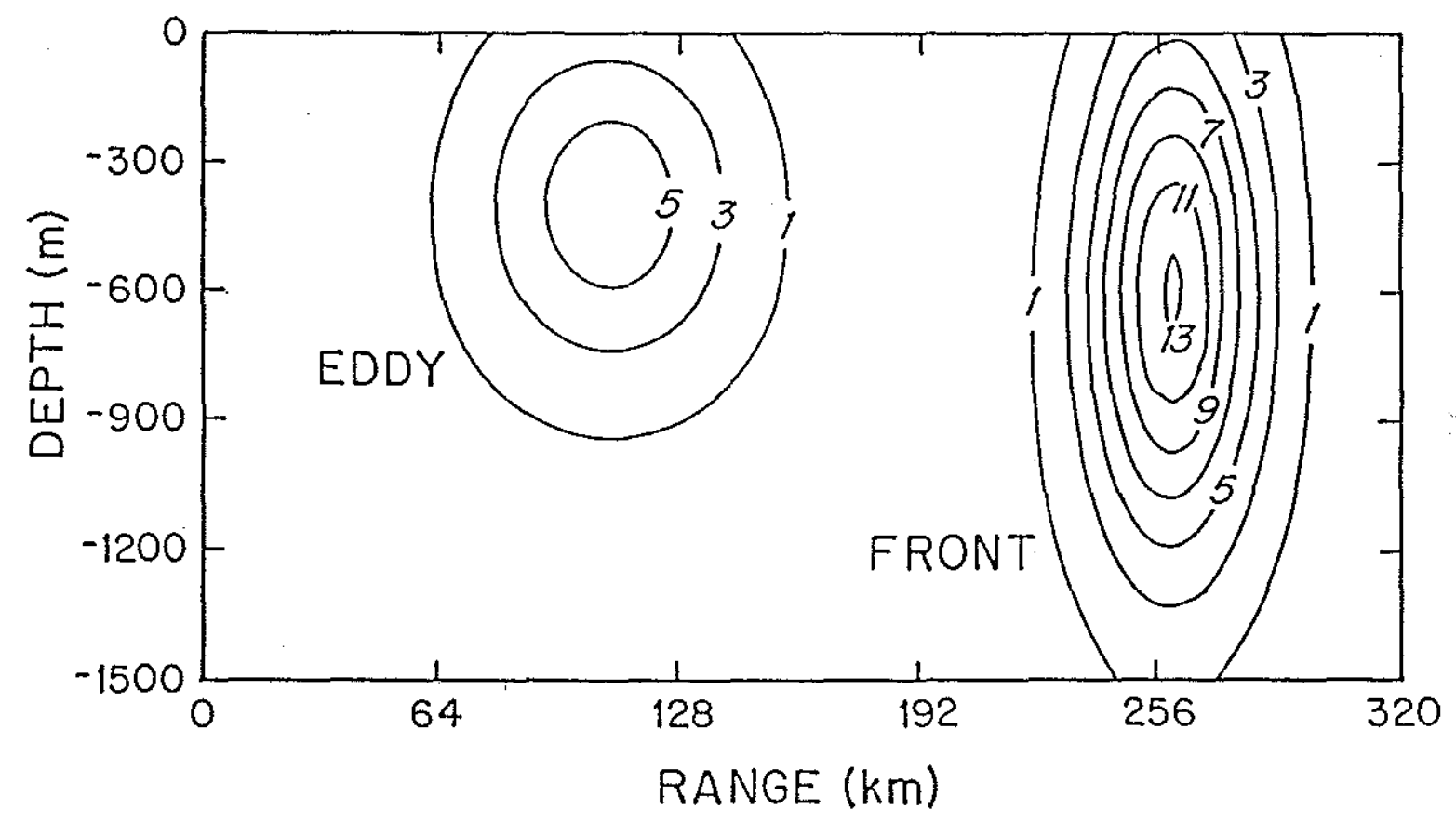

Figure 2-4: Vertical slice of input model for eddy and front, from $S 2$ to $R 1$ 
in the version of the three dimensional raytrace program we had at the time. HARPO is equipped to run certain canonical forms, like CBLOB3 (equation 2.10), to simulate sound speed perturbations. Therefore, the inverse sound speed perturbation results were fitted to equation 2.10. This fitting was done by measuring the distances between contours for each of the anomalies and extrapolating the average e-folding distance as well as the maximum sound speed increase for the eddy and front. The slice used for calculating the fit in depth and longitude was the path from source $S 1$ to receiver $R 2$ (figure 2-2 and figure 2-3). The slice from $S 2$ to $R 1$ was then used to extend the fit to three dimensions (figure 2-4) by indicating changes in sound speed perturbations in the latitudinal direction.

After the field reconstruction was completed, HARPO was used to send rays through the three dimensionally reconstructed ocean along the path from $S 3$ to $R 1$ (figure 2-2). This was done to examine the differences between rays going through the background, the model, the reconstruction without acoustics, and reconstruction with acoustics. Eigenrays were found and matched between these four cases. The important comparison characteristics are the arrival times and transmission loss (or intensity) of the eigenrays. Figure 2-5 shows a pair of rays separated by an angle $\Delta \theta$ traveling from the source $S$. The ray which leaves the source at angle $\theta$ is an eigenray for receiver $R$. Transmission loss due to geometrical spreading can be calculated for these rays in two dimensions using the following formula

$$
T L=10 \log \frac{\Delta A_{2}}{\Delta A_{1}}=\frac{R \Delta d}{\Delta \theta \cos \theta}
$$

where TL is transmission loss, $\Delta A_{2}$ is the area of the ray tube in the vicinity of the receiver, $\Delta A_{1}$ is the area at one meter, $R$ is the range from source to receiver, and $\Delta d$ is the perpendicular distance between the rays at the receiver. This method for computing transmission loss can be extended to three dimensions (Brekhovskikh and Lysanov, 1982) by treating variations in the plane where $z$ is constant in the same manner as the vertical plane. However, for our work, as will be shown in Chapter 3, the horizontal refraction effects on intensity were very small, so this two dimensional result was sufficient. 


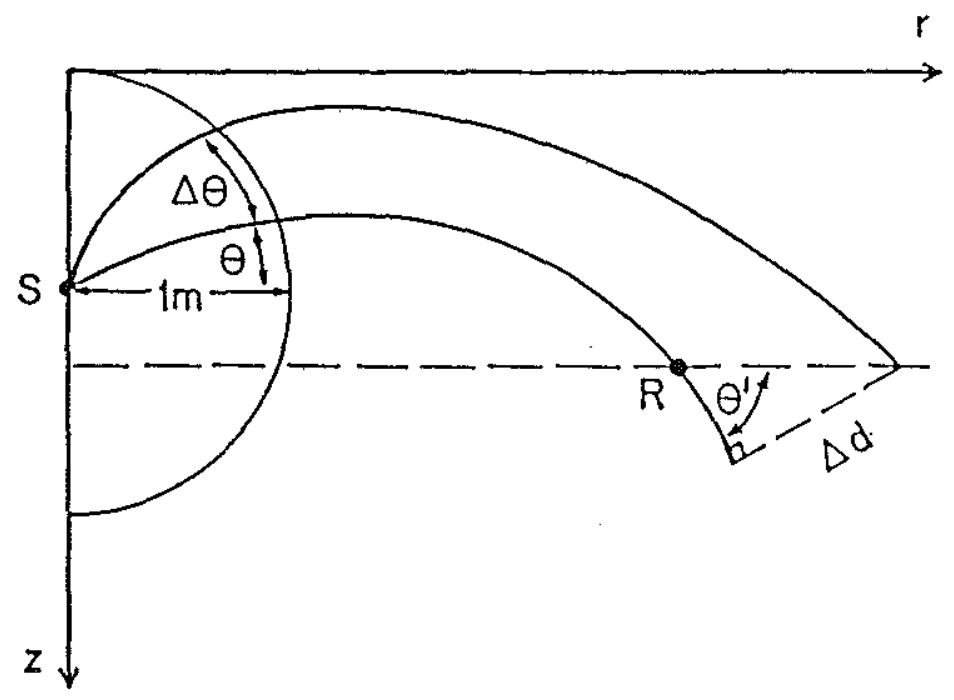

Figure 2-5: Diagram for calculation of transmission loss due to geometrical spreading 


\section{Chapter 3}

\section{Results and Analysis}

\subsection{Tomographic Reconstructions}

It is found that for our chosen ocean model, travel time differences between the background profile and the perturbed profile were between 60 and $150 \mathrm{~ms}$. The rays which remained identifiable were, for the most part, those which interacted with both the surface and bottom. This is a problem for vertical resolution, which is dependent on the turning points of rays. It can also create difficulties in receiving signals because transmission loss for rays which interact with the surface and/or bottom can often become large.

Nonlinearity due to strong perturbations can also cause inaccurate inversions. Figure 3-1 shows the paths for two eigenrays along path $S 2$ to $R 1$ which have approximately the same initial elevation angle. One ray goes through the background profile and the other through the perturbation. The perturbation has caused the raypath to change, especially at the range of the front, about $240 \mathrm{~km}$. Assuming the path of this ray to be frozen, i.e. the same for both the unperturbed and perturbed path, will lead to somewhat inaccurate results for a linear inversion. Another difficulty encountered is that with such strong perturbations, eigenrays which existed for the background ocean "disappear" in the perturbed model. Only rays which are clearly identified as stable can be used, as was previously mentioned.

Rays which interact with a flat surface and bottom have decreasing nonlinearity and the frozen path assumption is more nearly valid. They are also more stable and can therefore be identified for both the background and the perturbed sound speed profiles. In this work, 


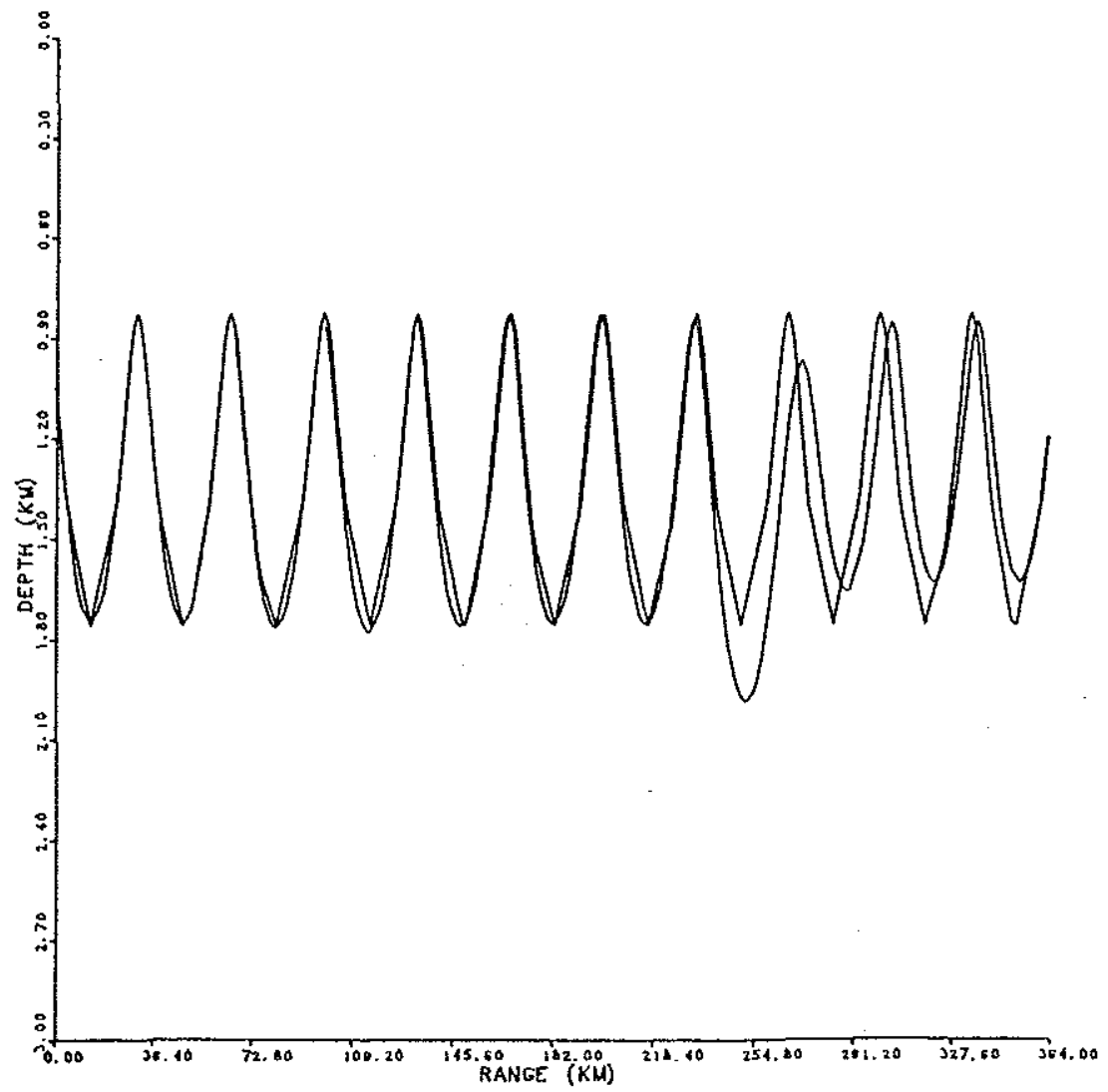

Figure 3-1: Background ray vs. perturbed ray 
27 rays were identifiable and stable for the path from $S 1$ to $R 2$ and of those, 23 interacted with both the surface and the bottom. For the path from $S 2$ to $R 1$ there were 36 stable rays and 31 interacted with the surface and bottom. The remaining rays still interacted with the bottom, but not the surface. Problems with identification were caused by the ray trace program showing several eigenrays with the same ray history, only slightly different elevation angles, but different travel times. Rather than using these rays, only those which were singularly identified were used. By using a background sound speed profile which is an average for the Gulf Stream region, the slowly increasing sound speed below the channel axis creates a channel which is very wide, so rays with lower elevation angles interact with the bottom. This will again cause reception difficulty over long ranges. Because this was a synthetic data set, bottom loss was not taken into account when choosing rays for the inversion.

In order to compute unique inversions, a priori information was provided. The diameter of Gulf Stream rings is approximately 100 to 200 kilometers and the vertical extent is from the surface to at least 750 meters depth (Lai and Richardson, 1977). Therefore, sound speed perturbations should be highly correlated within these distances. In order to be conservative in our estimates, the horizontal correlation length was specified as 50 kilometers and the vertical correlation length was 300 meters. In addition, the rms sound speed perturbation was $5 \mathrm{~ms}^{-1}$ and the $r m s$ experimental noise was $8 \mathrm{~ms}$. Sound speed perturbations were taken to be zero below 1500 meters depth and beyond 320 kilometers from the source.

The inversion for the path between $S 1$ and $R 2$, using "pure acoustics" (tomography only) is shown in Figure 3-2. Reconstructed anomalies are present and are fairly accurate in range, but not in depth or strength. The depth of the eddy is poor and its contours spurious. The strength of the front is not seen, nor is its deep vertical contours. In addition, there appears to be a great deal of noise, i.e. anomalies which do not exist. These are decreased by the amount of $r m s$ experimental noise included in the input which damps out noise contributions from other sources, but that value cannot be too great or travel time differences begin to lose their significance. In order to see more clearly the dimensions of features which can be adequately resolved by the "pure acoustics", Figure 3-3 indicates the horizontal and. vertical minimum resolution lengths. These show the size of the smallest features which 


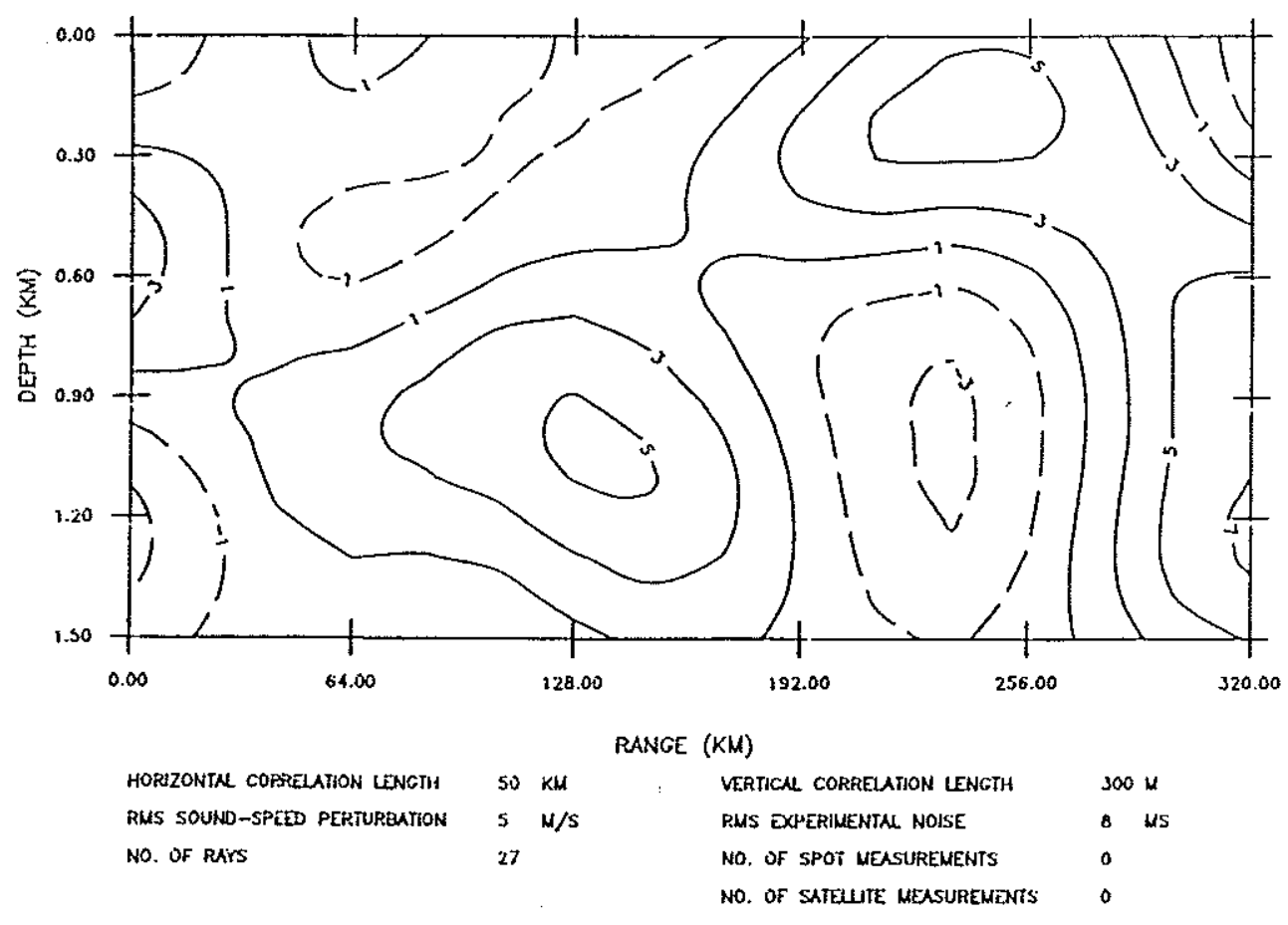

Figure 3-2: Sound speed perturbation estimate $\left(m s^{-1}\right)$ using "pure acoustics"

can be adequately estimated by the data provided (Chiu, et al., 1987). This figure shows that acoustics alone has poor vertical resolution and only marginally adequate horizontal resolution for anomalies located directly in the center of this area of the ocean. The fact that the rays used were mainly bottom interacting makes vertical resolution inadequate. Horizontal resolution is dependent on the spacing between ray crossings, which are the smallest halfway from source to receiver.

Adding satellite sea surface temperature data every 10 kilometers, with $.001 \mathrm{~ms}^{-1}$ sound speed error (i.e. negligible), produced the results shown in Figure 3-4. The result is still poor, showing a damping effect which removes some nonexistent anomalies, but no significant improvement in the eddy or front, indicating that more a priori information is needed. Previous work (Chiu, et al., 1987) showed that satellite data provided a great improvement to the acoustics. However, the anomalies treated were much smaller (approximately $4 \mathrm{~ms}^{-1}$ ) 


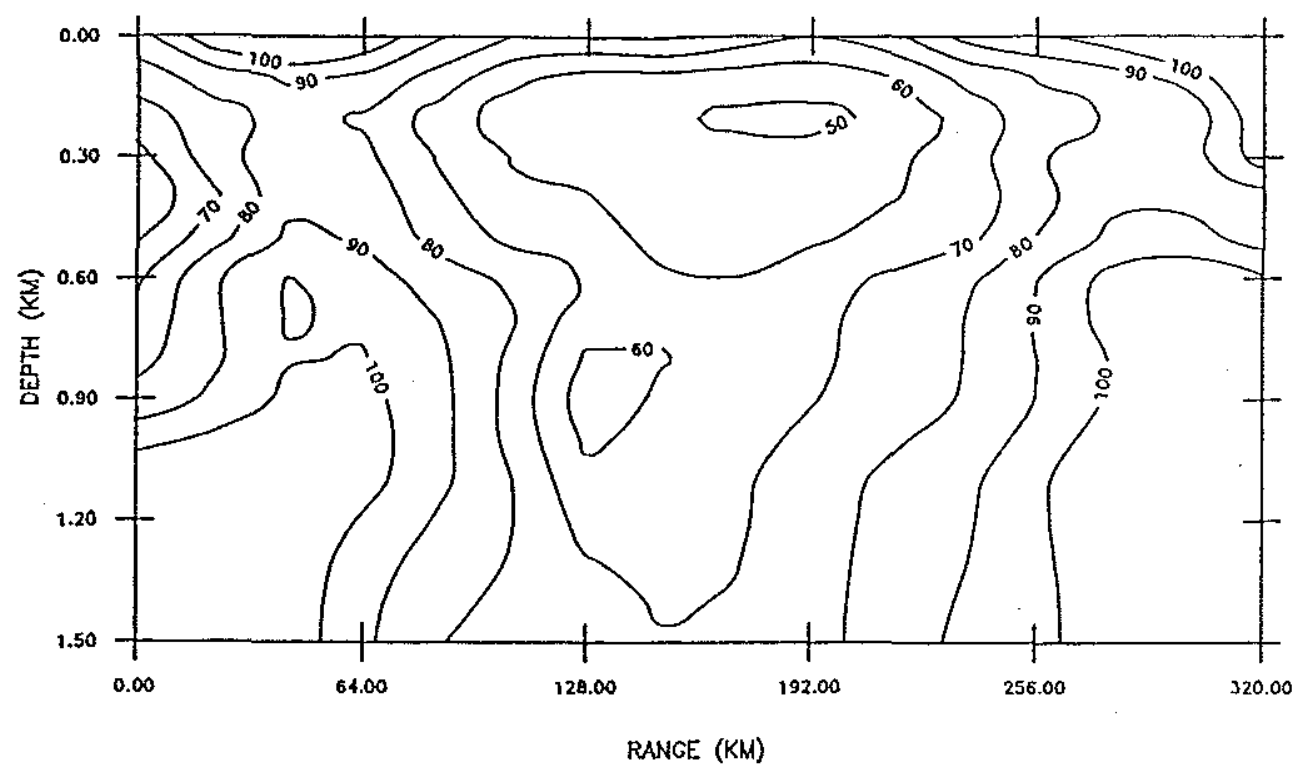

MINIMUM VERTICAL RESOLUTION LENGTH (M)

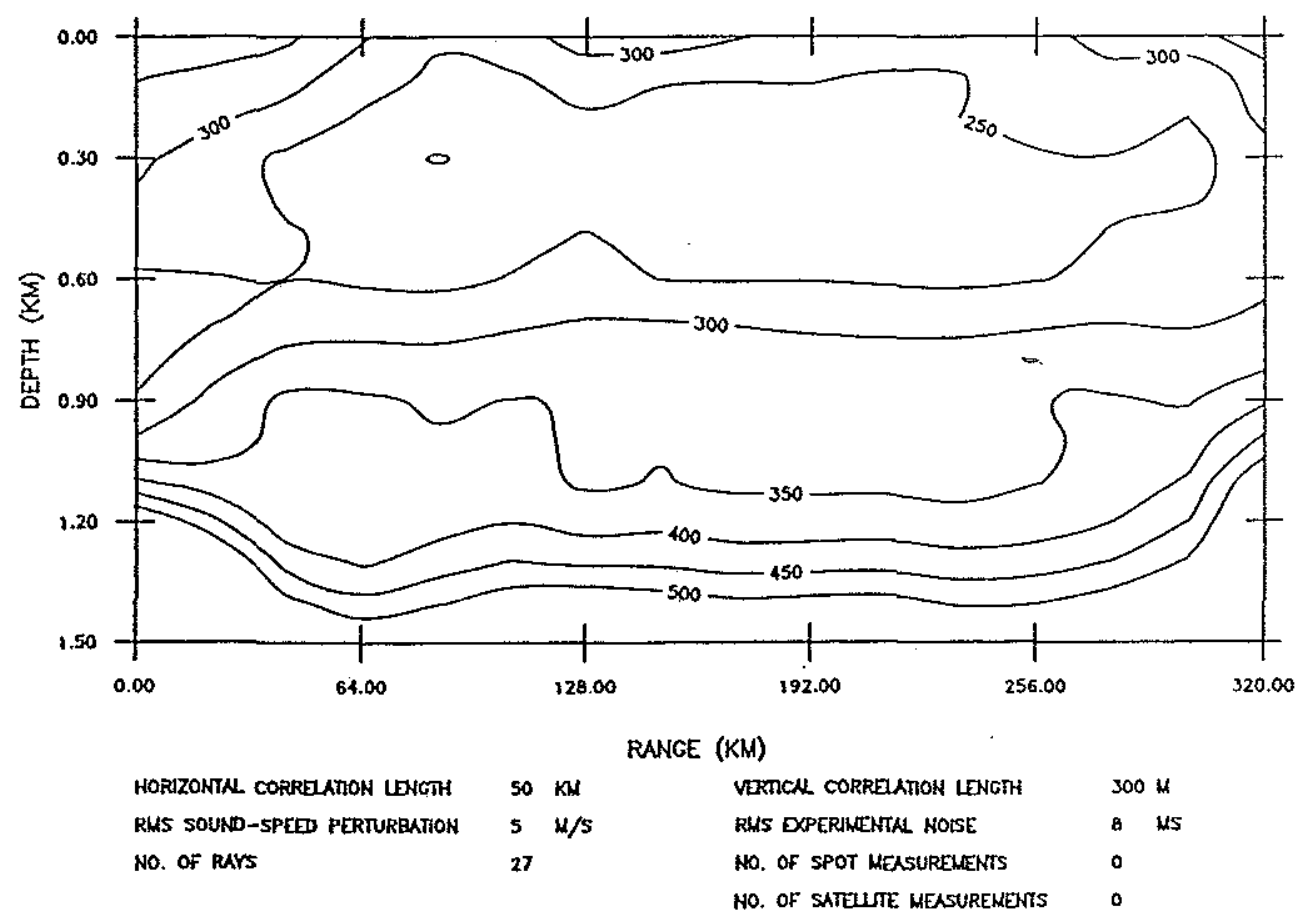

Figure 3-3: Horizontal and vertical resolution lengths for "pure acoustics" 


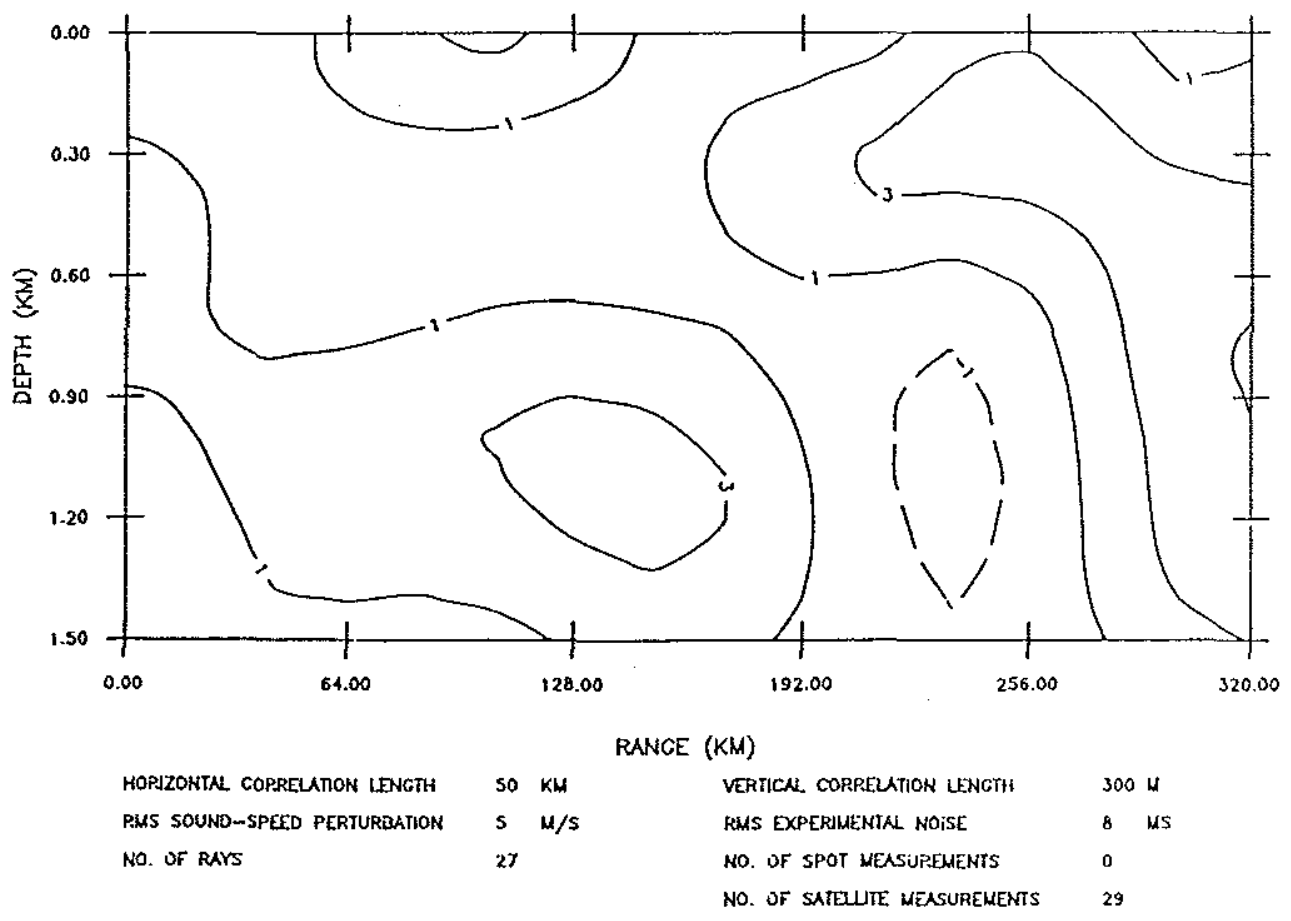

Figure 3-4: Sound speed perturbation estimate $\left(m s^{-1}\right)$ using acoustics and satellite sea surface temperature measurements

and fairly shallow. Figure 3-5 shows the horizontal and vertical minimum resolution lengths. These plots show expected improvement for the first 200 meters, but this does not improve the results because inadequacies in reconstruction are significant much deeper than this.

The inversions were next done using both acoustic data and XSV data with $0.5 m s^{-1}$ sound speed error. The result is shown in Figure 3-6, and shows enormous improvement. Figure 3-7 shows the resolution lengths for this case. Horizontal resolution is 50 kilometers down to approximately 750 meters, as opposed to approximately 250 meters in Figure 35. Vertical resolution does not show great improvement. Again, this is due to the use of bottom interacting rays.

Figure 3-8 shows the results for acoustics, satellite and XSV data combined. This shows some improvement from Figure 3-6 and is very good overall. The horizontal and 


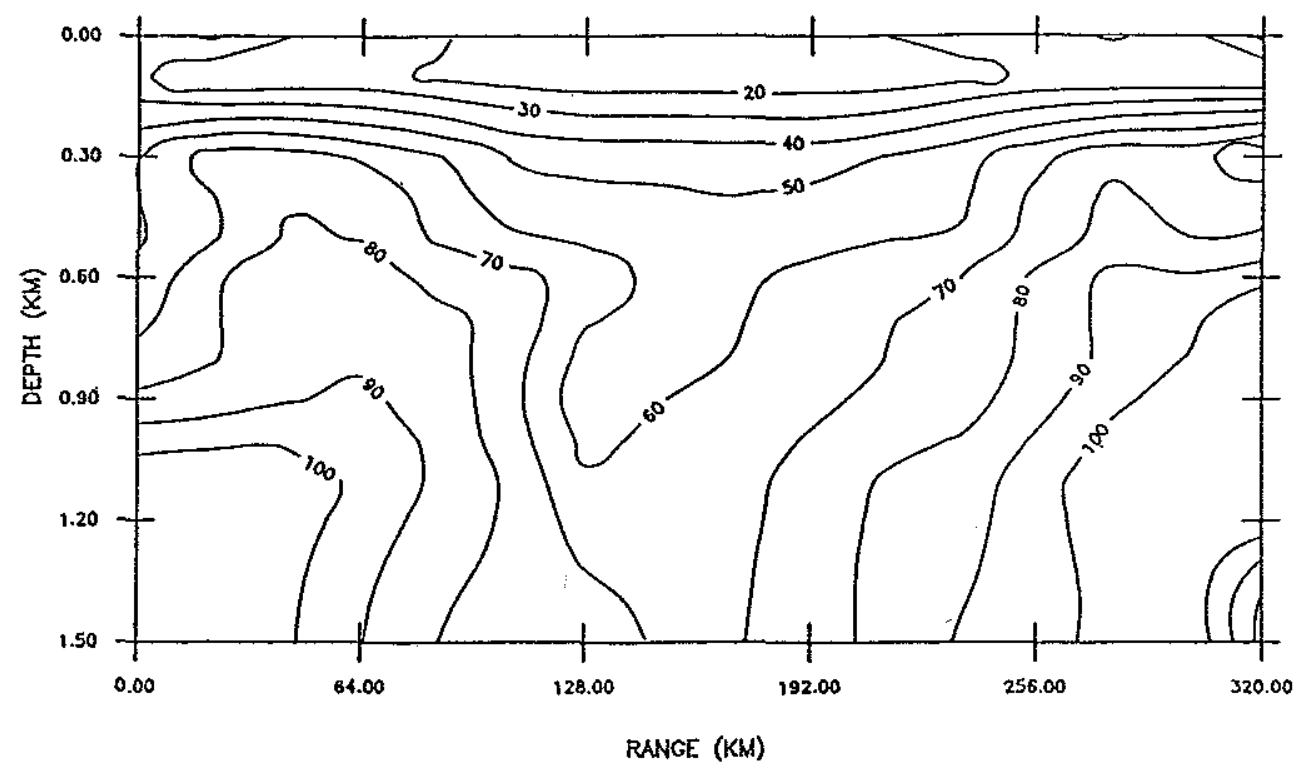

MINIMUM VERTICAL RESOLUTION LENGTH (M)

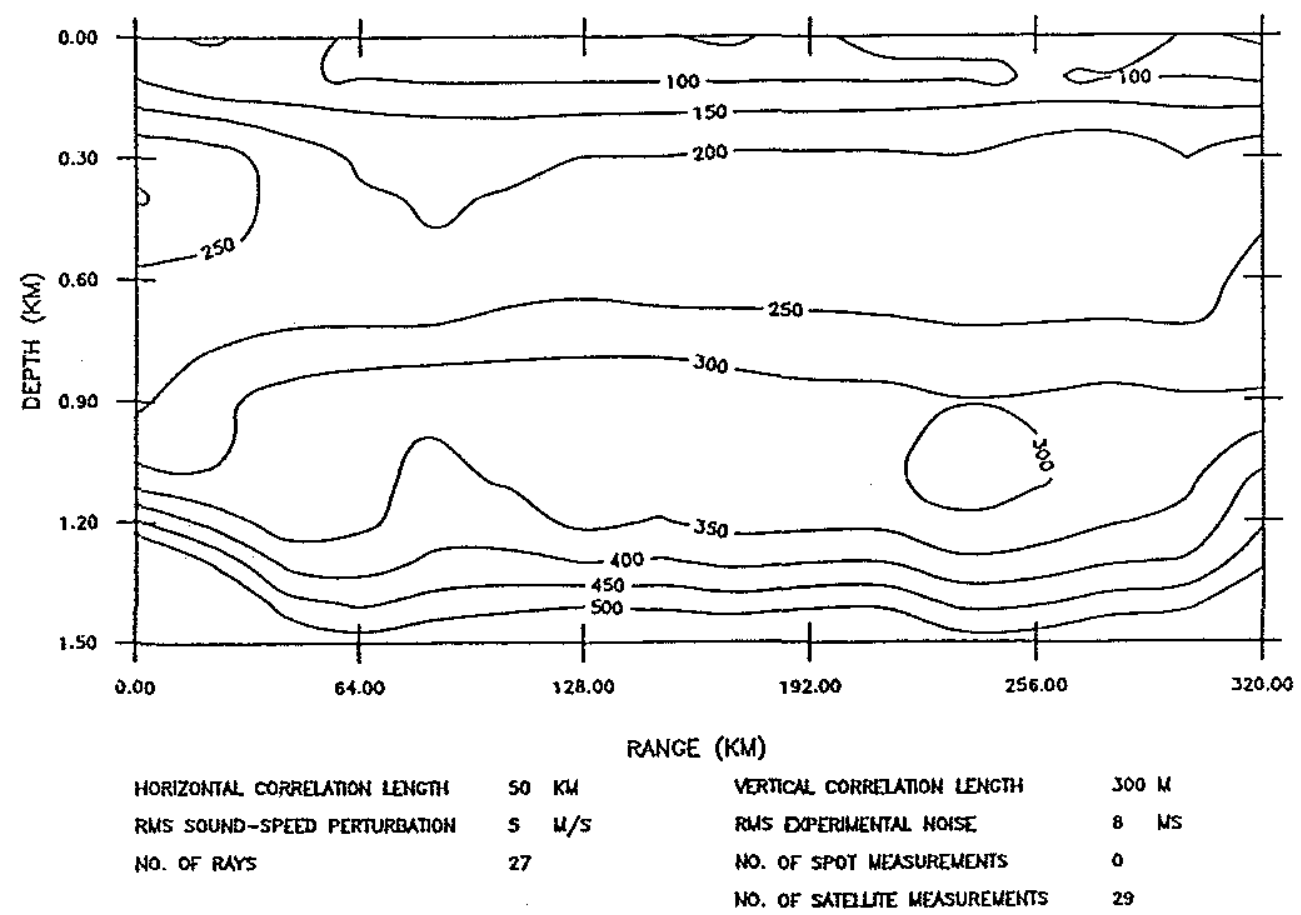

Figure 3-5: Horizontal and vertical resolution lengths for acoustics and satellite data 


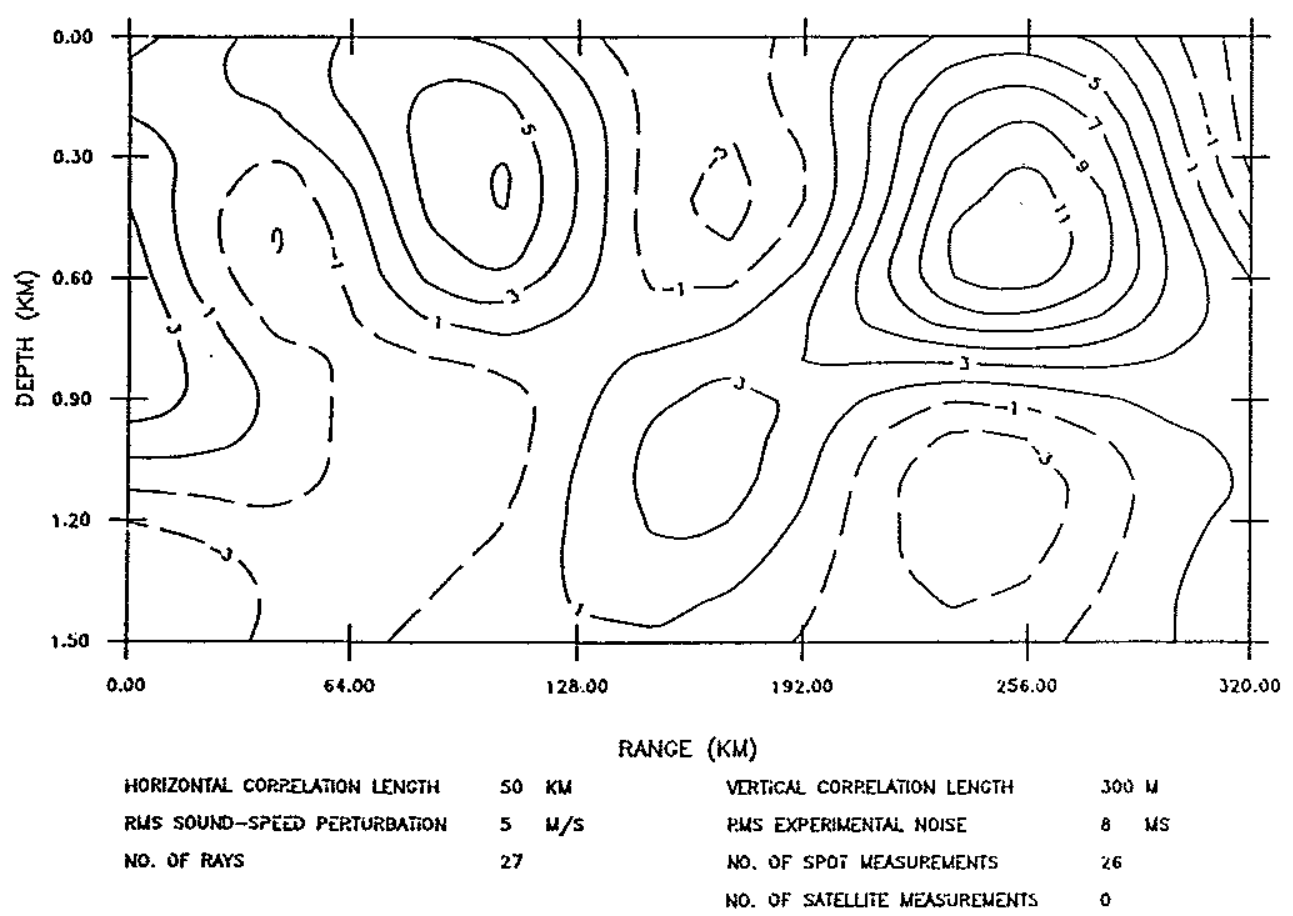

Figure 3-6: Sound speed perturbation estimate $\left(m s^{-1}\right)$ using acoustics and XSV data 


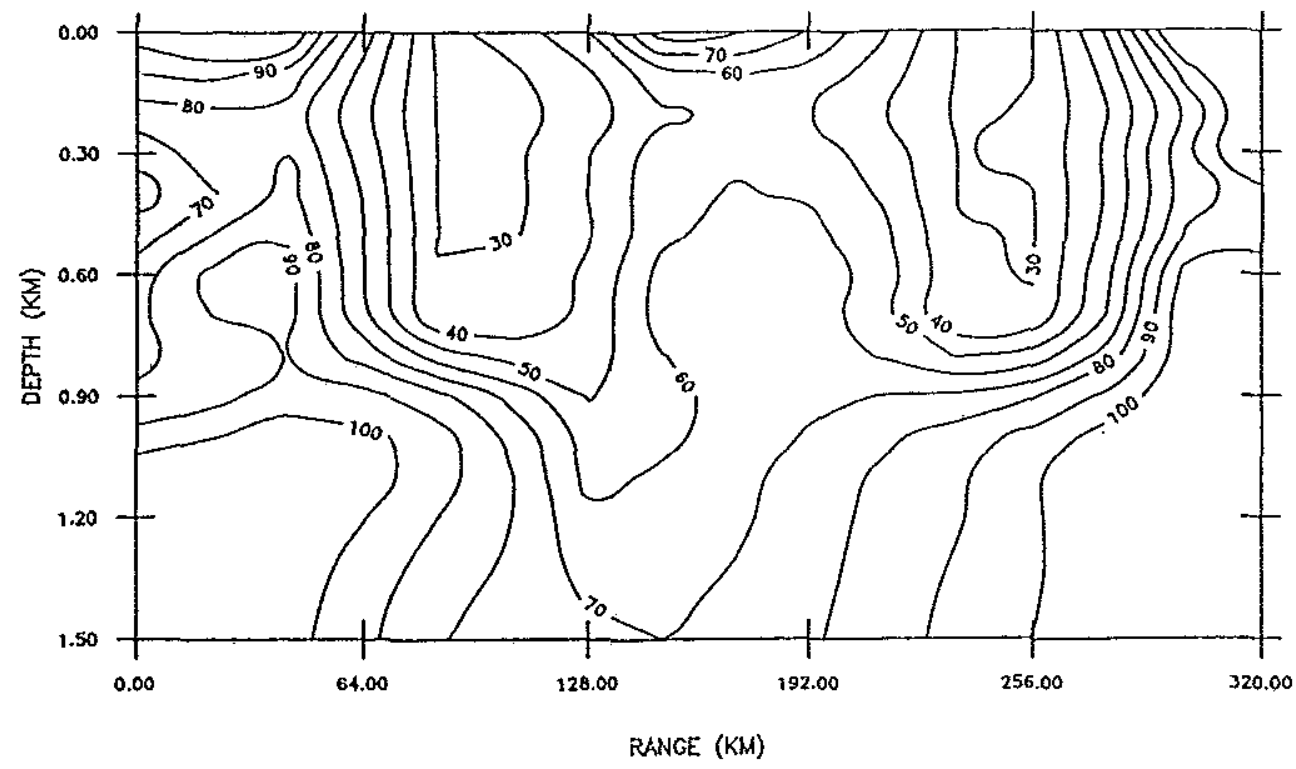

MINIMUM VERTICAL RESOLUTION LENGTH (M)

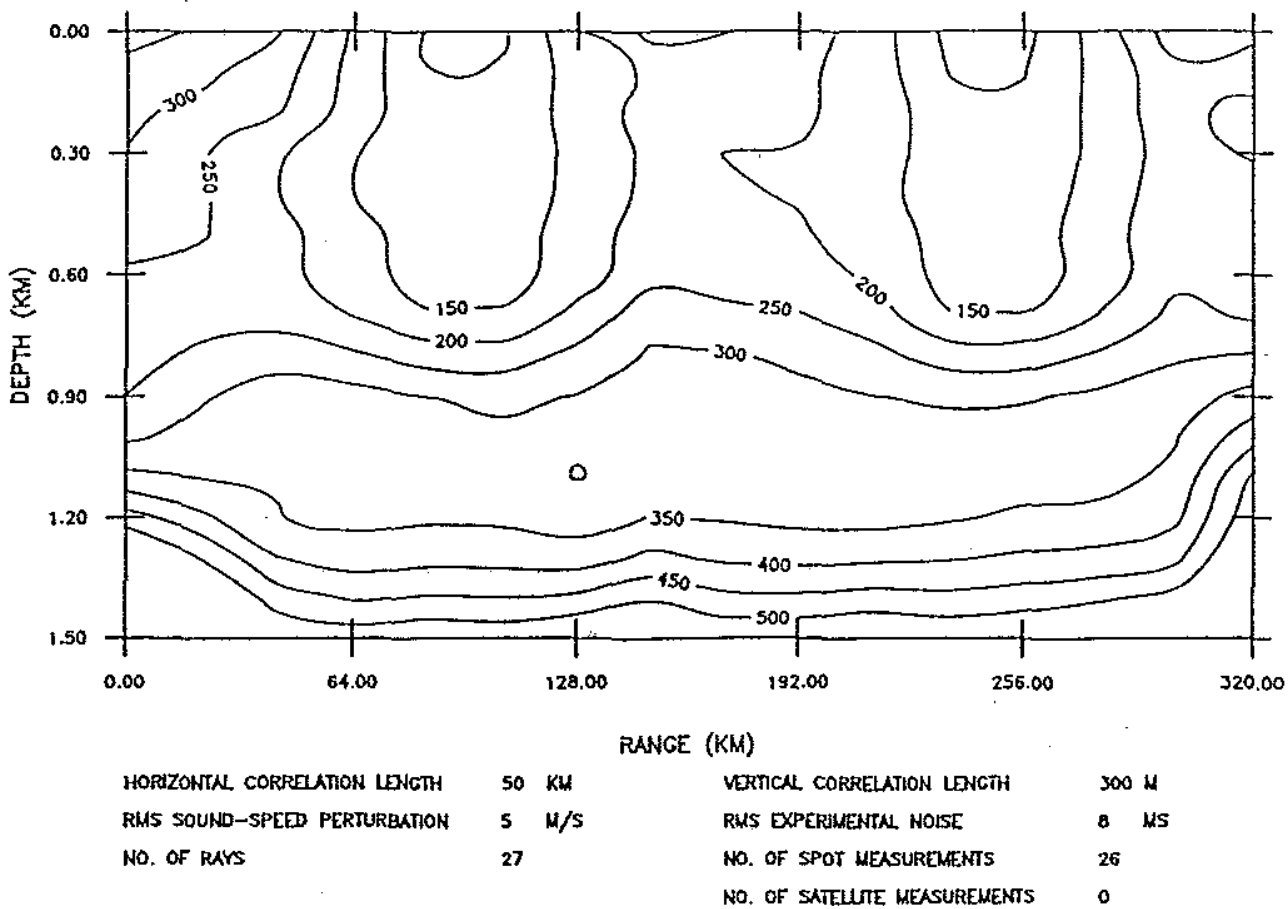

Figure 3-7: Horizontal and vertical resolution lengths for acoustics and XSV data 


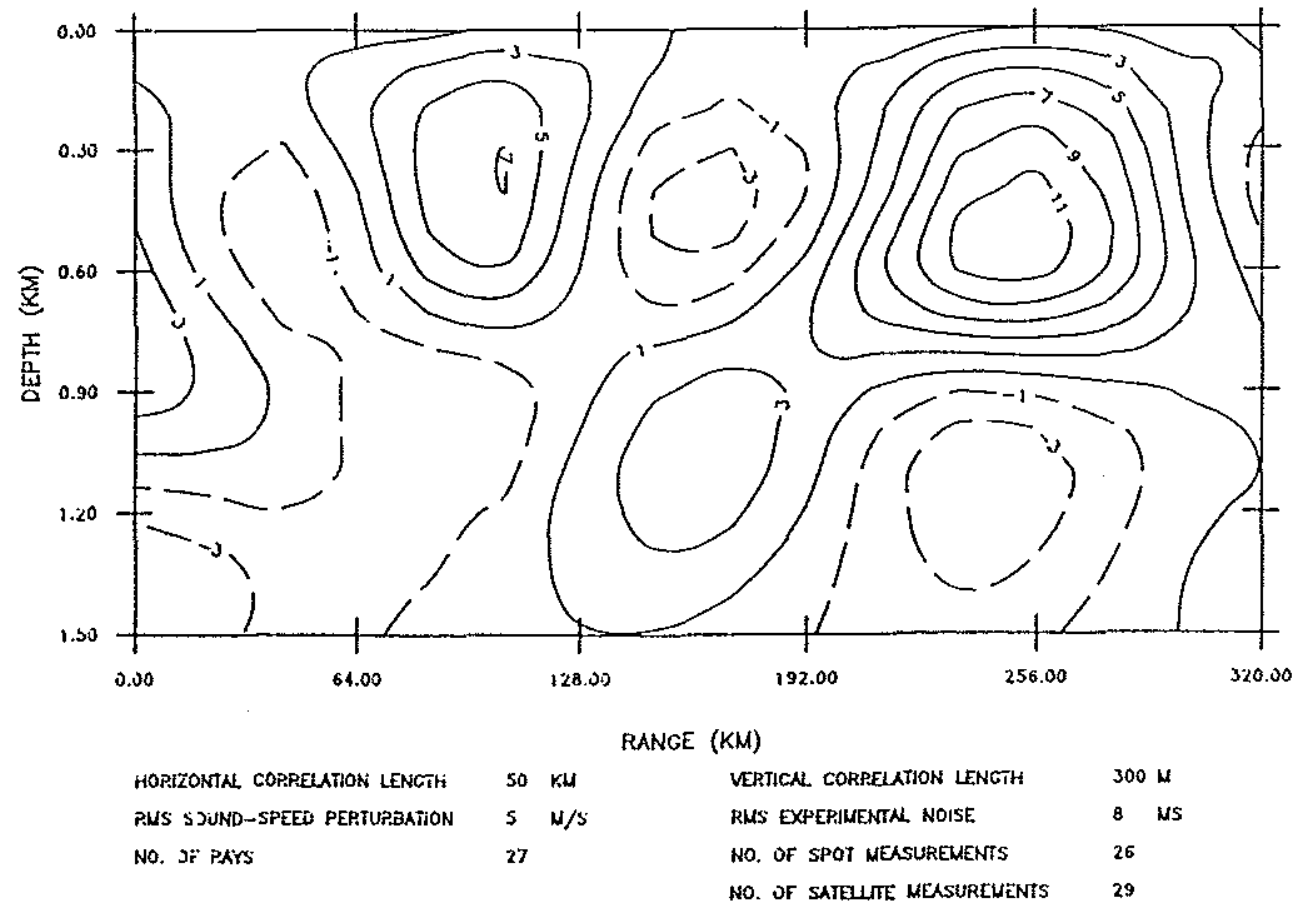

Figure 3-8: Sound speed perturbation estimate $\left(m s^{-1}\right)$ using acoustics, satellite and XSV data

vertical resolution lengths are shown in Figure 3-9. The improvement here, as contrasted with the previous figure, is at the surface, showing that satellite data in combination with XSV data provide a good complement to the acoustics data, which is often weak at the surface. Comparing Figure 3-8 to Figure 2-3 shows that we have done a creditable job of reconstruction.

In order to evaluate the benefits of acoustic data, an inversion was performed using XSV and satellite data, but no acoustics data. The result is shown in Figure 3-10. Figure 311 shows the resolution lengths for this case. It is clear that the shape of the anomaly, and how fast it decreases is caused by the choice of correlation lengths. Because our case was simple, this worked fairly well, but for a more realistic case, this type of inversion cannot work. Acoustics are needed to predict the shape and variability of the contours. 


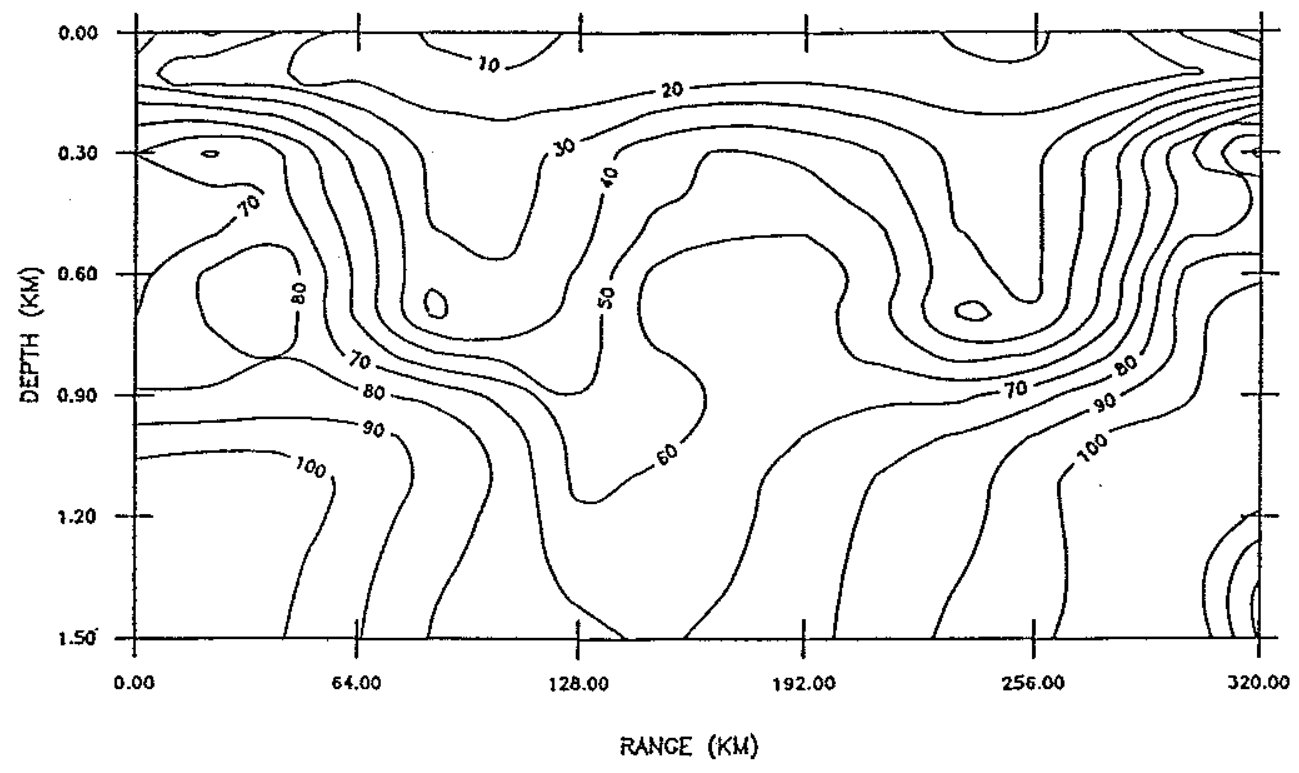

MINIMUM VERTICAL RESOLUTION LENGTH (M)

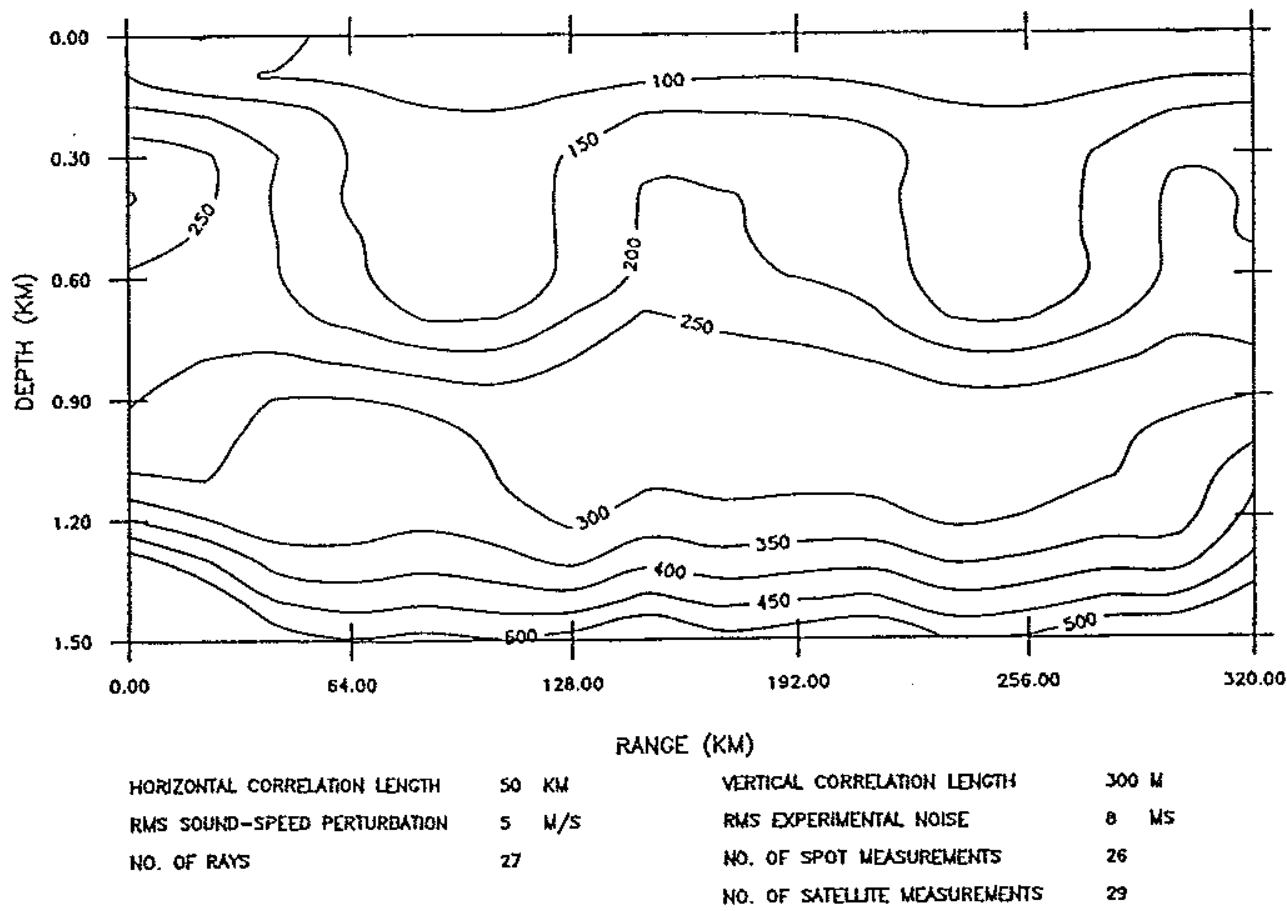

Figure 3-9: Horizontal and vertical resolution lengths for acoustics, satellite and XSV data 


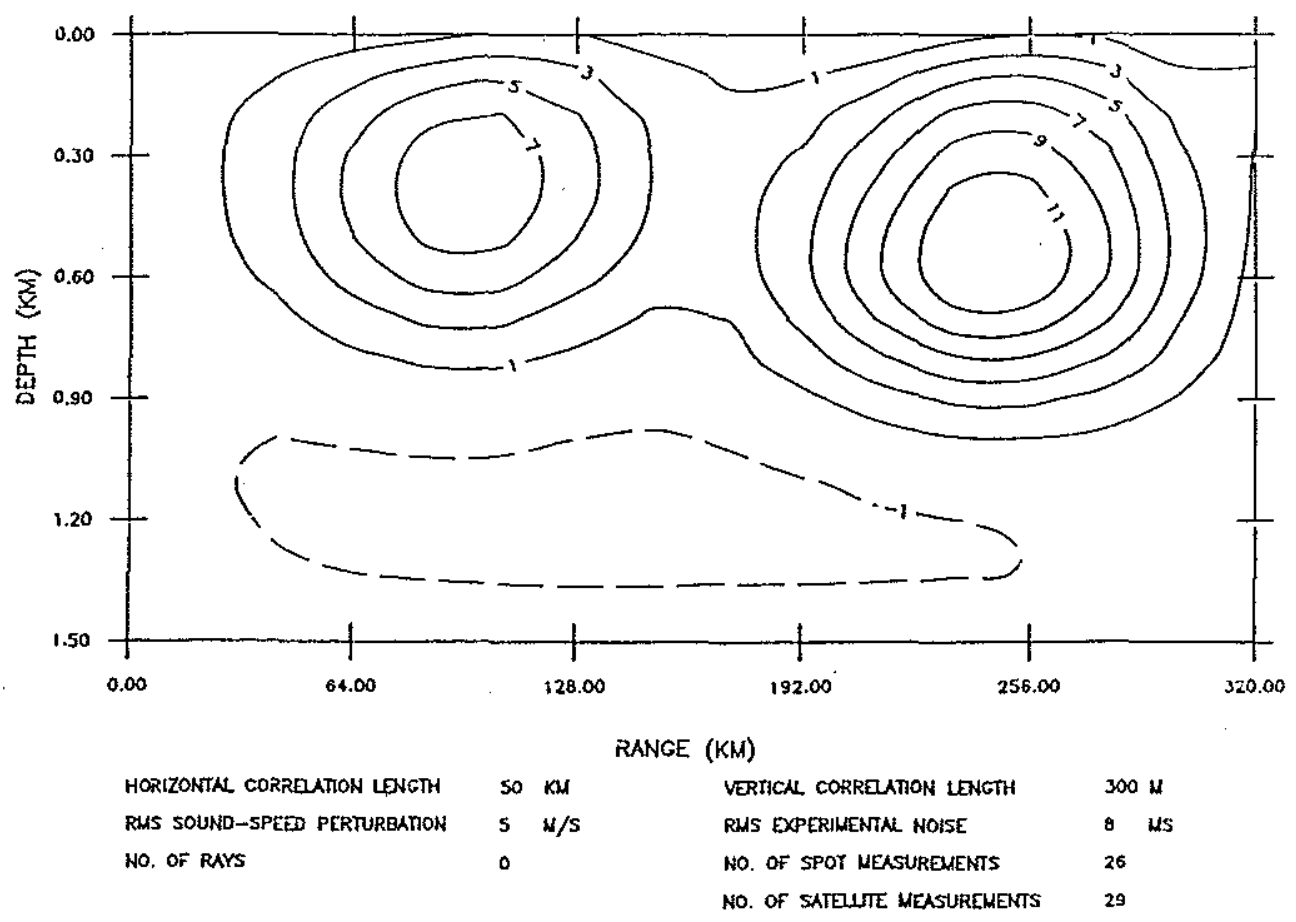

Figure 3-10: Sound speed perturbation estimate $\left(m s^{-1}\right)$ using XSV and satellite data, no acoustics

One can see in looking at the reconstructed anomalies, the case which uses acoustics has much better horizontal and slightly better vertical resolution. This means that unless XSV data is available continuously along the path being reconstructed, acoustics will provide a significantly better inversion. However, iterations are needed to accurately estimate the anomalies. In addition, more non-bottom interacting rays are needed to improve vertical resolution.

\subsection{Three Dimensional Propagation}

In order to test the results, the inversion estimates must be compared to the true model of the three dimensional ocean. As mentioned earlier, a fit of the inversion results to equation 2.10 can be used to run three dimensional rays through a volume of ocean using 


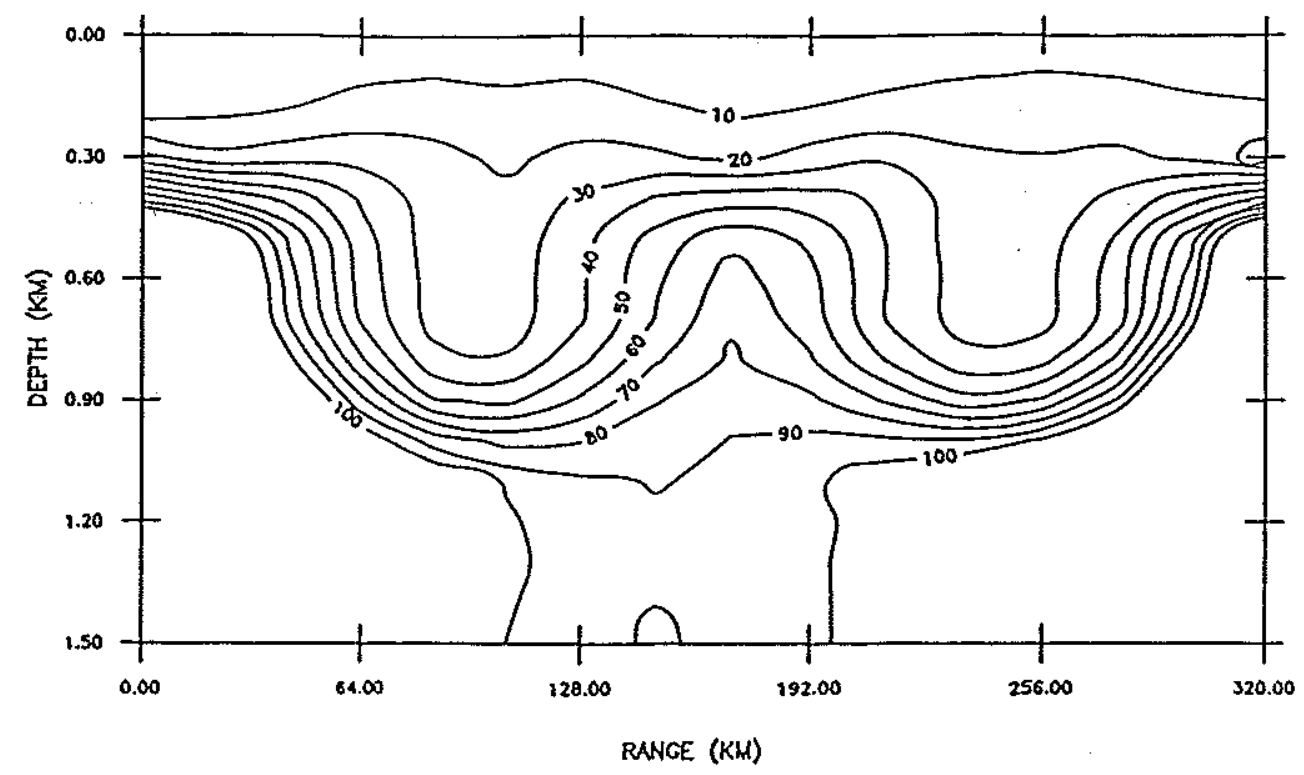

MINIMUM VERTICAL RESOLUTION LENGTH (M)

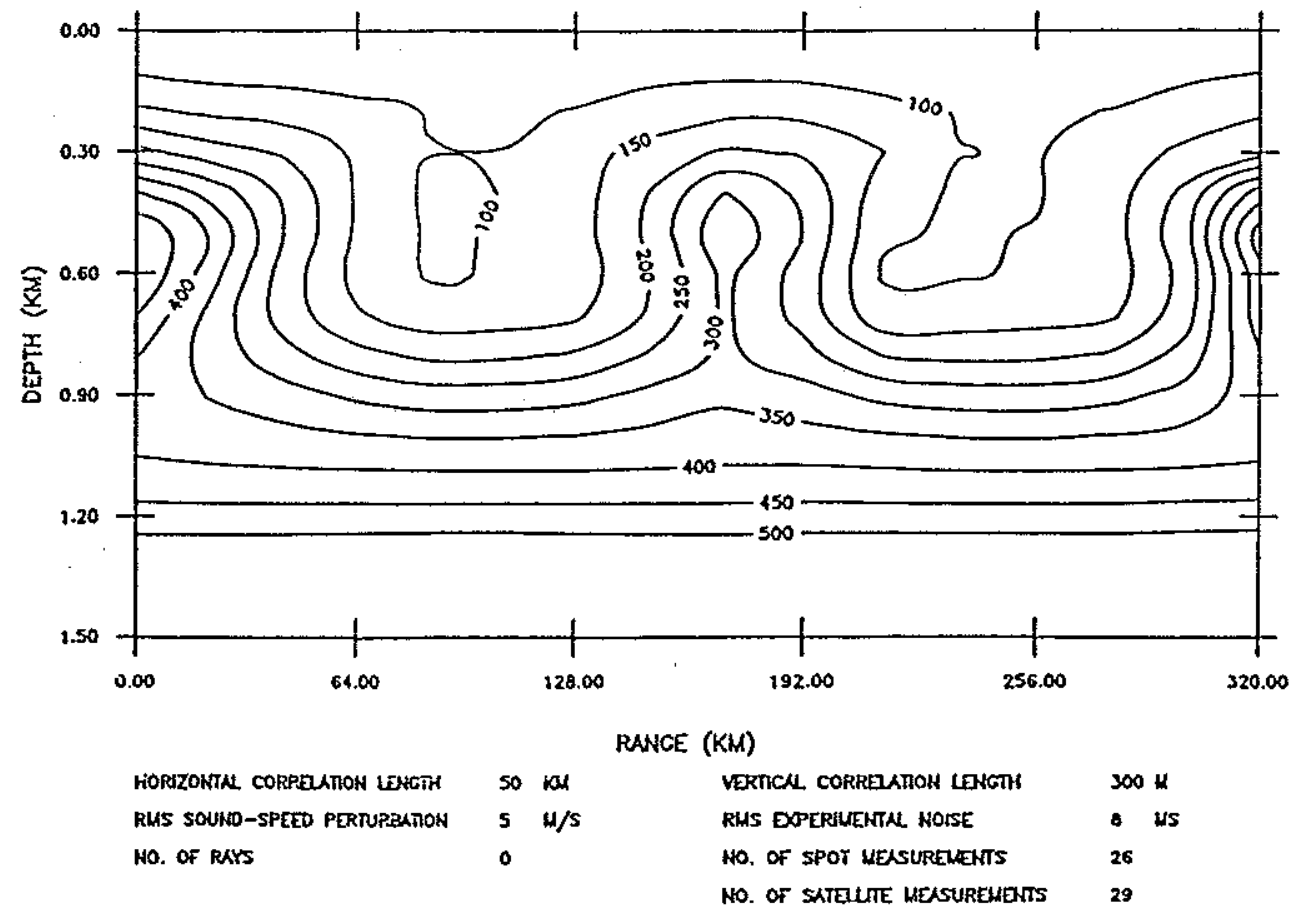

Figure 3-11: Horizontal and vertical resolution lengths for XSV and satellite data, no acoustics 


\begin{tabular}{||l||l||l|l|l|l|l|l|l||}
\hline \multicolumn{1}{||}{} & CASE & $\Delta_{i}$ & $\begin{array}{l}W_{z} \\
(\mathrm{~km})\end{array}$ & $\begin{array}{l}W_{\theta} \\
(\mathrm{km})\end{array}$ & $\begin{array}{l}W_{\phi} \\
(\mathrm{km})\end{array}$ & $\begin{array}{l}z_{i} \\
(\mathrm{~km})\end{array}$ & $\begin{array}{l}\theta_{i} \\
(\mathrm{~km})\end{array}$ & $\begin{array}{l}\phi_{i} \\
(\mathrm{~km})\end{array}$ \\
\hline \hline \multirow{3}{*}{ EDDY } & Model & .0067 & .395 & 33.0 & 33.0 & .4 & 100.0 & 100.0 \\
\cline { 2 - 9 } & $\begin{array}{l}\text { Inverse } \\
\text { (w/acoustics) }\end{array}$ & .0066 & .284 & 29.0 & 24.0 & .345 & 100.0 & 99.3 \\
\cline { 2 - 9 } & $\begin{array}{l}\text { Inverse } \\
\text { (w/o acoustics) }\end{array}$ & .0076 & .283 & 35.9 & 44.4 & .361 & 100.0 & 97.6 \\
\hline & $\begin{array}{l}\text { Model } \\
\text { Inverse w/acoustics } \\
\text { (w/acoustics) }\end{array}$ & .0091 & .384 & 0.0 & 41.7 & .518 & 100.0 & 249.3 \\
\cline { 2 - 9 } & $\begin{array}{l}\text { Inverse w/o acoustics } \\
\text { (w/o acoustics) }\end{array}$ & .0097 & .418 & 0.0 & 45.2 & .506 & 100.0 & 243.4 \\
\hline
\end{tabular}

Table 3.1: CBLOB3 (equation 2.10) data for model, inversion with acoustics, and inversion without acoustics

HARPO. This fitting was done by measuring the distance between contours and calculating the gradients for a Gaussian model. Using these gradients, the sound speed perturbations at the center of the anomalies were extrapolated outwards and values of the fractional increase in sound speed were computed. The values used for the true model, the inverse with acoustics, XSV and satellite data, and the inverse without acoustics for equation 2.10 are listed in Table 3.1. These figures show that the acoustics inversion has recreated an ocean with smaller sound speed perturbations, but these extend appropriately to greater depths than the inversion without acoustics. Figure 3-12 shows graphically the Gaussian fit of the inversions with acoustics for the path from source $S 1$ to receiver $R 2$ (see Figure 2-2 and Figure 2-3). Again, the sound speed perturbations are smaller, but conform better to the shape of the true model. Iterations of this inversion would produce results which were closer to the model, but for a first iteration the result is adequate. (Problems with resolution below 750 meters depth would not greatly improve, however. Sources and receivers would need to be located deeper to improve resolution at these depths). The inverse result for this same slice using XSV and satellite data, but without acoustics is shown in Figure 3-13. The values at the center of the anomalies are closer, but the shape is laterally stretched across the ocean area. If the anomalies we modeled were more irregular, this inversion would have shown greater inadequacies. Moreover, there is no further iteration possible using this data. 


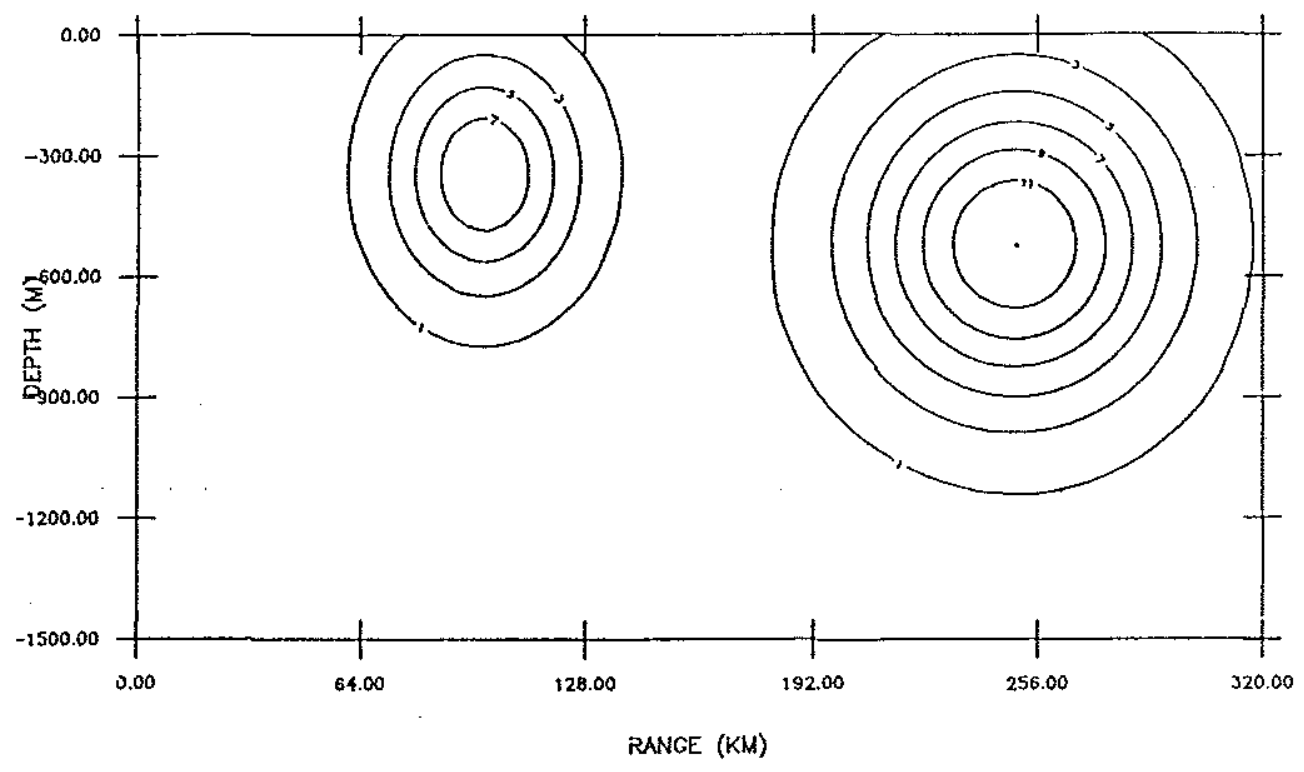

Figure 3-12: Gaussian fit to inversion with acoustics, path $S 1$ to $R 2$

We should also note that we have optimized this reconstruction by putting XSV's in the center of the features. Placing the XSV's anywhere else would have given an inferior result to the one shown.

Rays were then propagated along a third path, from $S 3$ to $R 1$ (see Figure 2-2). The purpose of this was to examine the changes in signals expected at the receiver between the background profile, the model, the inversion with acoustics, and the inversion without acoustics, for a completely different path than those used for the inversion, but originating within the reconstructed volume. Using HARPO, rays were propagated through this path and eigenrays calculated. These eigenrays were identified by their ray histories and compared between the four cases. Rays between angles $-15^{\circ}$ and $+15^{\circ}$ were used, less rays than for the original inversion to avoid rays which interact with the bottom many times. Using the model as the standard, 36 eigenrays were found. After computing the transmission loss 


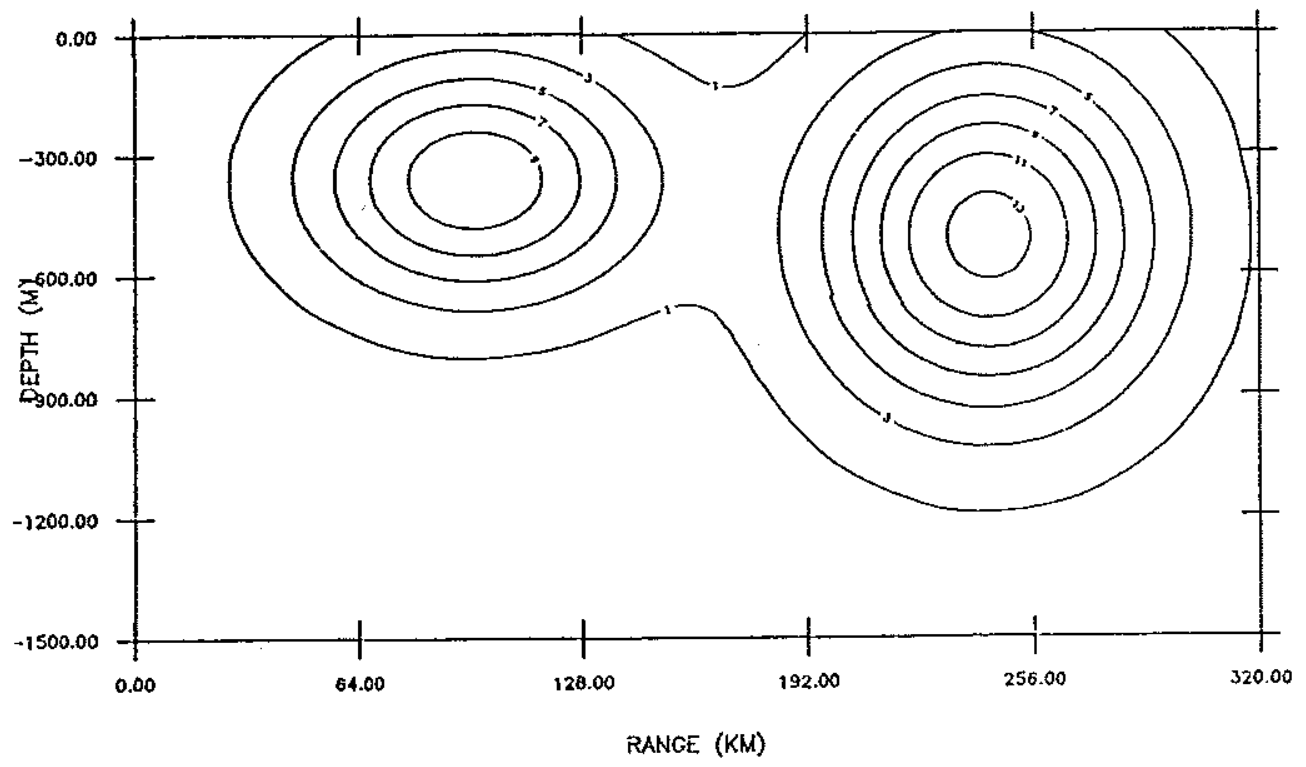

Figure 3-13: Gaussian fit to inversion without acoustics, path $S 1$ to $R 2$ 
for each of these, only those with losses of less than $115 \mathrm{~dB}$ were used. For the model, there were 21 of these rays and 14 could be matched to eigenrays for the other three cases. This again indicates the problem with stability encountered when trying to identify eigenrays. The code used to find eigenrays, however, solved for them uniquely by tracking ray histories so ambiguity between rays for each case were not encountered. Figure 3-14 shows the changes in raypath between an eigenray going through the background profile and one with the same ray history going through the model. The greatest change in the path is encountered in the later portion when the model eigenray passes through the front. This same ray type through the model and the inversion with acoustics is shown in Figure 3-15. There still appears to be a great difference in paths through the front, but the two rays are very close for their path through the eddy. Again, for strong perturbations, like the front, nonlinearities will cause the inversion to be less exact. Iterations would help to make these results more accurate. Finally, Figure 3-16 shows the results for the same ray type through the model and through the inversion without acoustics. The result is very similar to that found using acoustics. However, with acoustics, the match to the model for the eddy is slightly better and one does not see the ray affected by the front until later in its trajectory. Again, this points to the need for iterations of the acoustic inverse for the front in order to dampen out the effects of nonlinearities.

In Chapter 2, the method for calculating transmission loss due to geometrical spreading was shown. It was stated that horizontal effects were so small that the two dimensional approach was adequate. Its computational simplicity made this a desirous method for doing these calculations. In order to show that the three dimensional effects are small, Figure 3-17 shows the vertical and horizontal paths for two eigenrays through the model. The eigenray which does not interact with the bottom is the same ray used in the last examples. In the horizontal view, it has the smallest deflection. In order to have it intersect the receiver, the azimuth angle was decreased by $.023^{\circ}$, a very small amount. Consequently, displaying these rays in the horizontal for all three cases showed no significant difference in the results. The second ray, at a larger angle shows greater deflection. Even so, its deflection requires a change in azimuth angle of only $.084^{\circ}$, which is still very small. Transmission loss can therefore be calculated in two dimensions without significant error. 


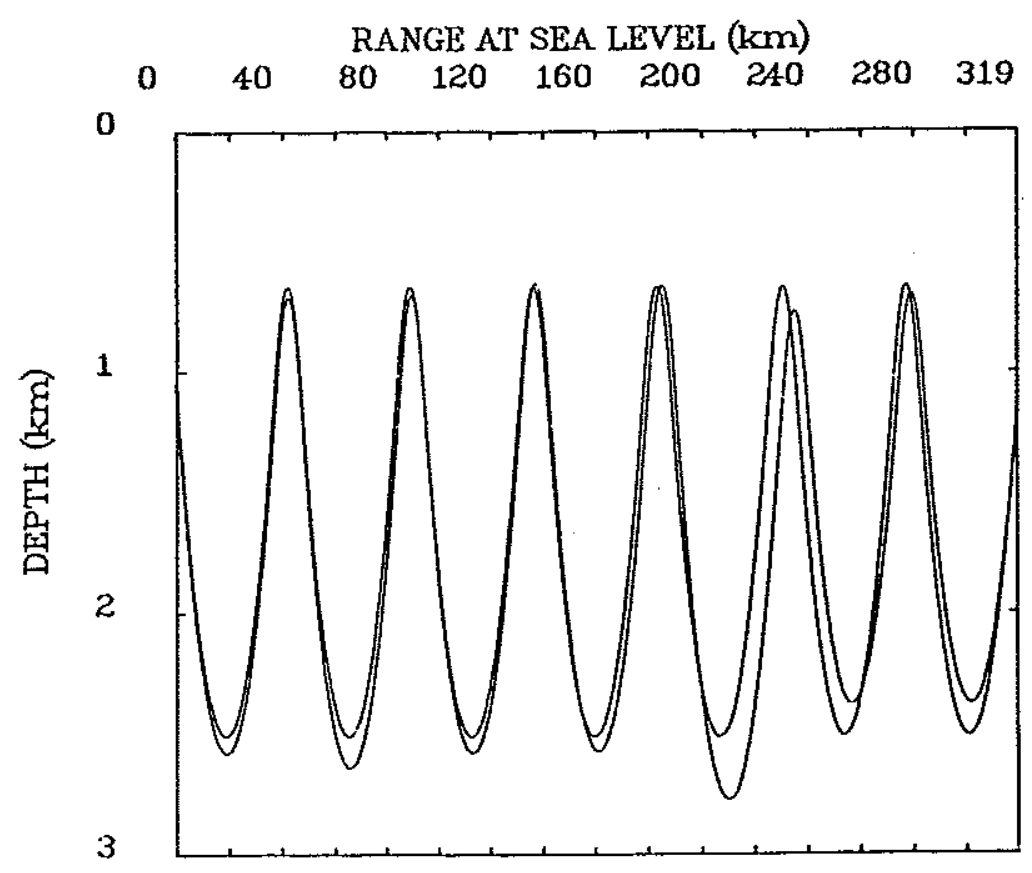

Figure 3-14: Model vs. background raypaths 


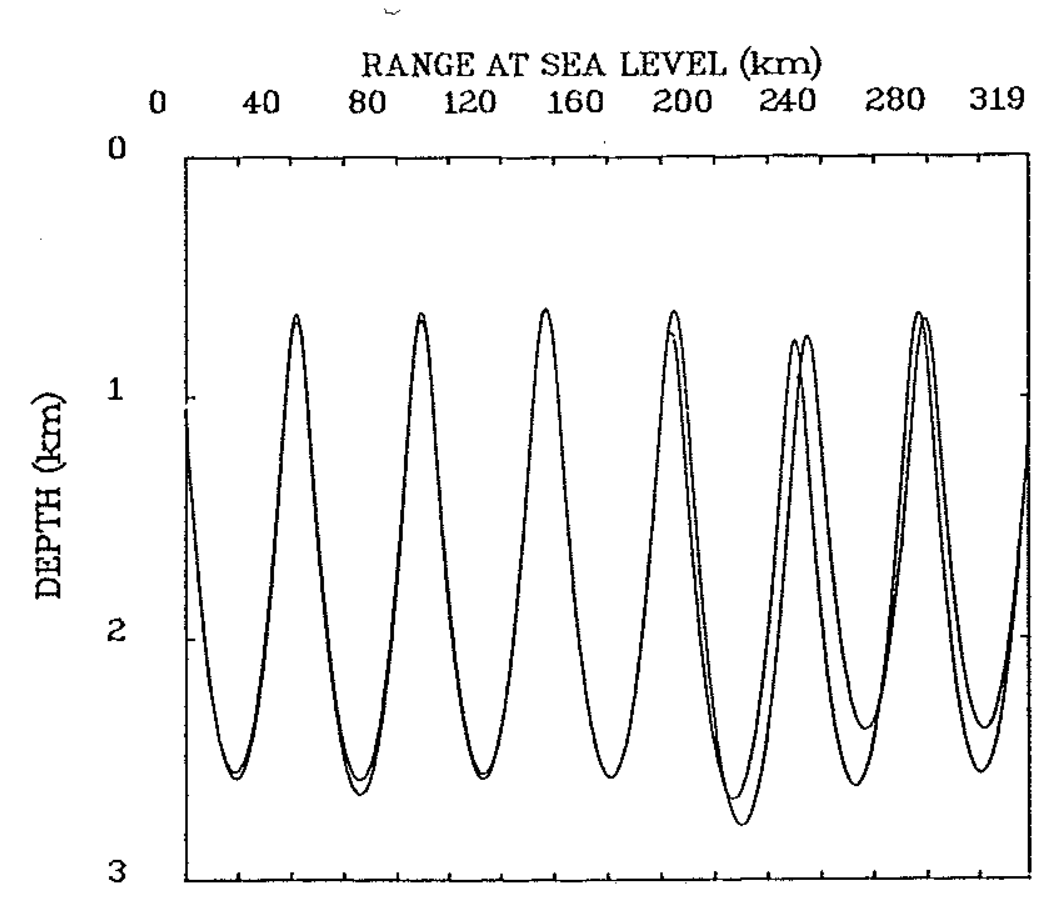

Figure 3-15: Model vs. inverse with acoustics raypaths 


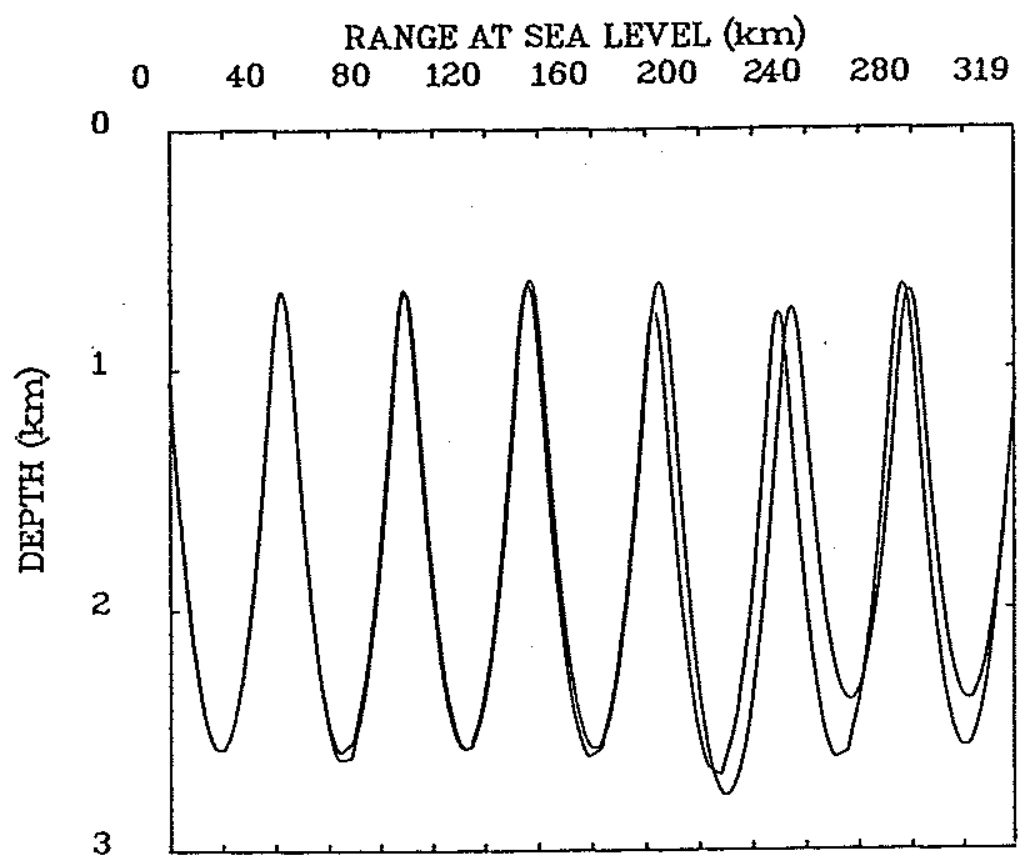

Figure 3-16: Model vs. inverse without acoustics raypaths 

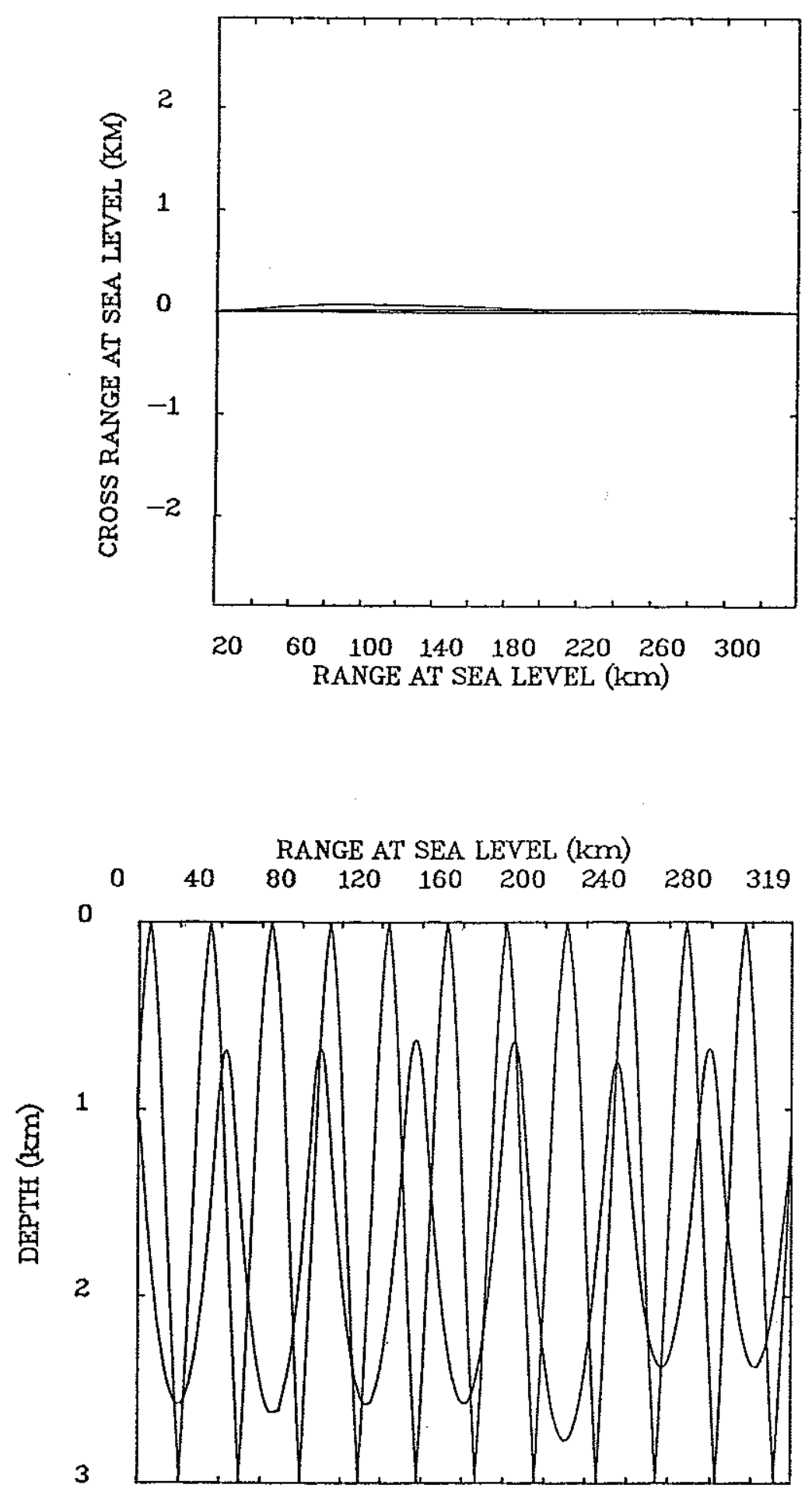

Figure 3-17: Two rays through the model, horizontal and vertical aspect 
Figures 3-18 and 3-19 show the transmission loss versus time for the 14 identified rays for the four cases. The sign in front of the identification numbers next to each line indicates whether the ray had an up or downgoing initial elevation angle. The identification number itself indicates how many turning points there were. It can be seen that between the model and the background, there is translation in arrival time for the eigenrays. Rays with the same history arrived $60 \mathrm{~ms}$ or more faster than the same rays in the background. This is due to the increase in sound speed within both the eddy and the front. One can also see the large transmission loss for two eigenrays in the background case, which have low losses for the model. This is less likely for a background which is close to the "real ocean". This effect can be seen in the two inversion cases, which do not have rays which have such large transmission loss. The plot of the inverse with acoustics shows again a translation in time, but much smaller. For the early arrivals, this translation is approximately $5-16 \mathrm{~ms}$. Later arrivals are shifted up to $53 \mathrm{~ms}$. Transmission losses are very close. The plot showing the results for the inversion without acoustics also shows fairly good results. Early arrivals are very close in time (less than $14 \mathrm{~ms}$ ) and in transmission loss. A late arrival has a high value for transmission loss, again indicating disparity in the inverse and the model. The last two . arrivals are in opposite places for this result, the +16 ray being the last arrival for the model and the -22 ray being the last arrival for the inverse without acoustics data. Transmission loss for each might identify this switch in a field experiment, but more than likely these arrivals would not be identified. 

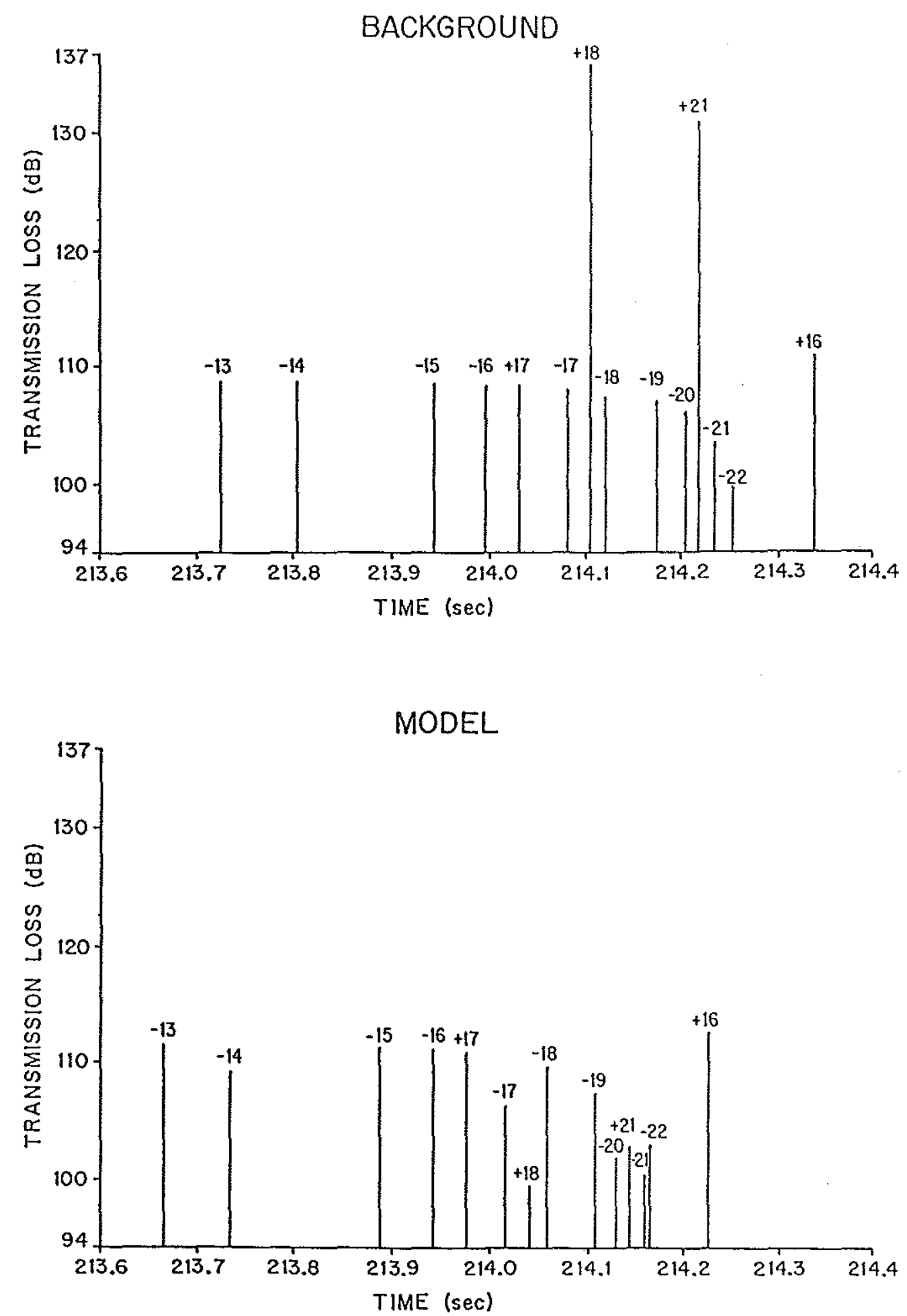

Figure 3-18: Transmission loss versus time of ray arrivals for the background profile and the model 

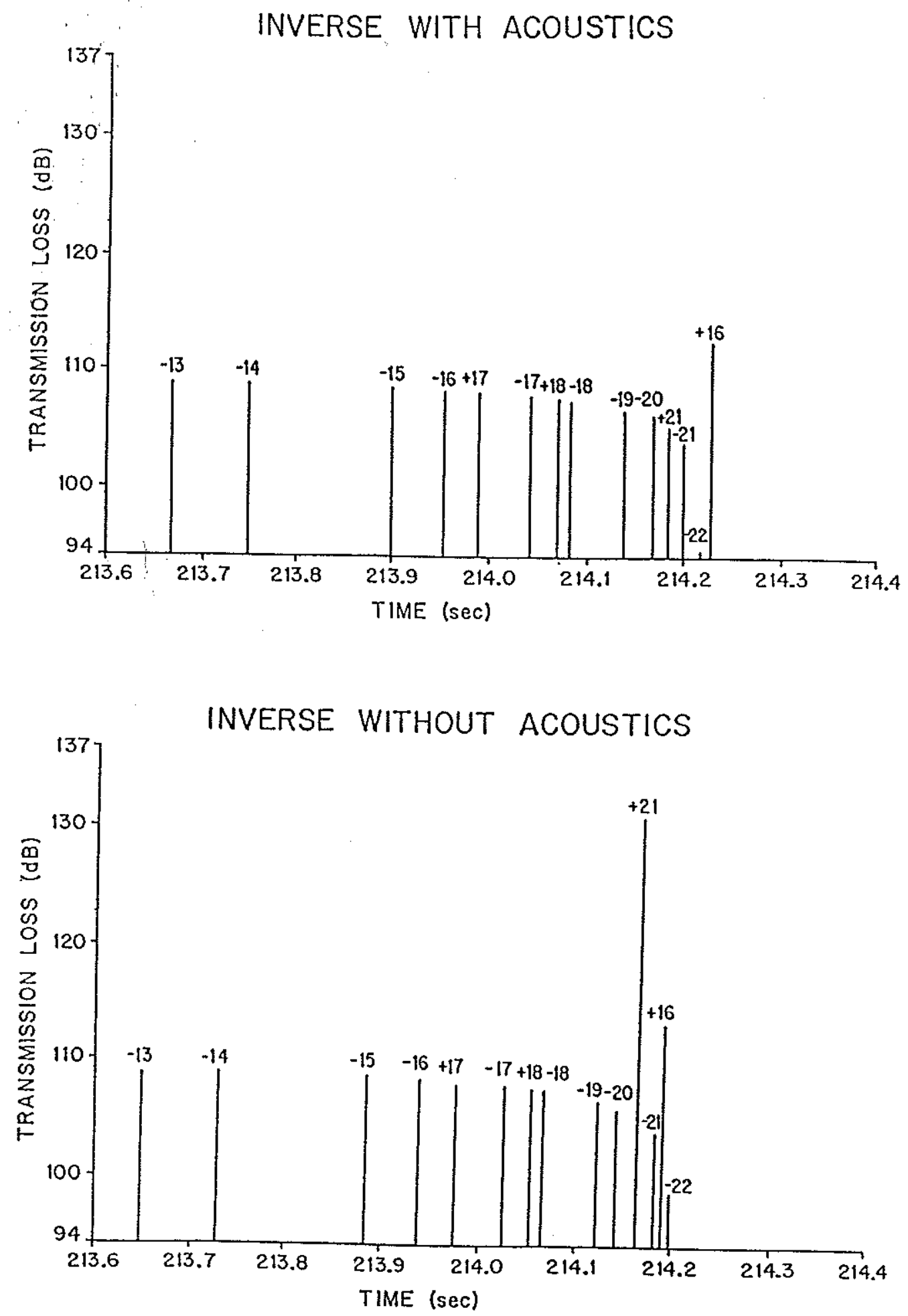

Figure 3-19: Transmission loss versus time of ray arrivals for the inversion with acoustics and the inversion without acoustics 


\section{Chapter 4}

\section{Conclusions}

Ocean acoustic tomography has been shown effective in reconstructing the (synthetically. modeled) three dimensional ocean volume, but must be aided by XSV and satellite sea surface temperature data to reconstruct very strong sound speed perturbations. For shallow perturbations, satellite data have been shown effective, but for anomalies (such as those modeled) which are deep, solutions are not adequate without XSV data. In addition, iterations are required in the acoustics to reduce nonlinearities caused by strong sound speed perturbations. The presence of a background profile which more closely matches the anomalies would therefore yield better results. More purely refractive rays would also improve results. Without these rays vertical resolution is poor and the reconstructed anomalies do not extend to the proper depths, falsely compensating for this in the horizontal direction by "smearing out" the contours. In the Gulf Stream region, the average sound speed profile has a SOFAR channel which is very wide. However, the strong sound speed perturbations of the Gulf Stream can almost make the channel disappear. Therefore, rays with low elevation angles go very deep, often interacting with the bottom. In order to have refractive rays, then, elevation angles must be shallow; however these rays are more unstable than steep rays. Therefore, refractive rays are more difficult to identify and use for inversions. However, a more realistic case with water depths up to 5000 meters would yield more refractive rays, improving vertical resolution.

Using only the XSV and satellite sea surface temperature data, without acoustics data, the inversion results were surprisingly good. However, the anomalies were spread across the 
ocean area, joining in the center. The XSV data were taken directly at the center of the eddy and front. If the XSV data were not taken at the center of the anomalies, the maximum anomaly would have been located at the point where the data was taken, yielding poorer results. In our calculations, the data used for the inverse included the correlation lengths and data variance. These were determined, because this was a synthetic case, by a priori knowledge. When data is very limited, as it was for this case, these a priori statistics govern the inverse. Consequently the results were as predicted by the covariance function. When more data is available, the data will govern the inversion. This is the case when using acoustic tomography, so the results depend on what was sampled rather than what was known about the area before the inversion was done. In areas like the Gulf Stream system, which experience large changes in short time periods, tomographic results are needed to provide accurate three dimensional pictures.

Propagating three dimensional rays through the Gaussian fits extrapolated from the inversions showed the usefulness of the method. Ray intensity stick plots showed very similar results between the model and the acoustical inversion. Using this first inversion as the background model for a second iteration would yield even better results. Eigenrays propagated through the reconstructed field showed little horizontal deflection. A Gulf Stream model which had more realistic temperature and current effects, as well as varying topography, would perhaps show more horizontal deflection, though results to date show that only topography produces very large deflections (Lynch, private communication). The small amount of horizontal deflection for eigenrays in our work shows that source horizontal bearing $(\phi)$ estimates using conventional beamforming techniques should be only slightly improved by the reconstructions. The variance in eigenray structure between the original model and the reconstruction is, in this case, not crucial for the bearing estimation. For matched field processing, however, which localizes the source in range, horizontal angle, and depth $(r, \phi, z)$, eigenray structure is crucial. Comparing the eigenray intensity and travel time results between the model and the reconstructions shows, just by inspection, that the reconstructions are not good enough to support matched field processing. However, our results are not the best reconstructions of the ocean which can be done.

Due to time and program development limits, certain procedures could not be done for 
this work which would have improved results. First, a full three dimensional inversion program was not available, so we were forced to use the vertical slice inversions. This method is suboptimal. However, a three dimensional inversion program has now become available. Until very recently, eigenrays could not be traced through arbitrary features using HARPO, so reconstructed ocean features had to be fit to canonical features which HARPO could use. Presently, using empirical orthogonal functions (EOF's) or ocean quasi-geostrophic (QG) modes, HARPO can accomodate any ocean sound speed or current structure desired. Ocean models like the Harvard Open Ocean Model can also aid studies such as ours by providing realistic synthetic data. These areas should be explored. Given these improvements, perhaps the reconstructions could be improved so as to be useful for matched field processing, depending on how strong the sound speed perturbations are. Attempting to perform matched field processing with HARPO's eigenray finder, though difficult and timely, would then be possible. Presently, HARPO can be used to identify eigenrays for many source/receiver positions, a procedure necessary to complete this process. 


\section{Bibliography}

Brekhovskikh, L. M., 1960. Waves in Layered Media. Academic Press, New York.

Brekhovskikh, L. M. and Yu. Lysanov, 1982. Fundamentals of Ocean Acoustics. Springer-Verlag, New York.

Chiu, C. S., J. F. Lynch and O. M. Johannessen, 1987. Tomographic Resolution of Mesoscale Eddies in the Marginal Ice Zone: A Preliminary Study. J. Geophys. Res., 92, $\mathrm{C} 7,6886-6902$.

Clay, C. S. and H. Medwin, 1977. Acoustical Oceanography: Principles and Applications. John Wiley and Sons, Inc., New York.

Cornuelle, B., 1982. Acoustic Tomography. IEEE Transactions on Geoscience and Remote Sensing, GE-20, 3, 326-332.

Cornuelle, B., 1983. Inverse Methods and Results from the 1981 Ocean Acoustic Tomography Experiment. Ph.D. thesis, Massachusetts Institute of Technology/Woods Hole Oceanographic Institution, $359 \mathrm{pp}$.

Cornuelle, B., C. Wunsch, D. Behringer, T. Birdsall, M. Brown, R. Heinmuller, R. Knox, K. Metzger, W. Munk, J. Spiesberger, R. Spindel, D. Webb, and P. Worcester, 1985. Tomographic Maps of the Ocean Mesoscale, Part 1: Pure Acoustics. J. Phys. Oceanogr., 15, 133-152.

Cornuelle, B. and P. Malanotte-Rizzoli, 1986. A Maximum-Gradient Inverse for the Gulf Stream System. J. Geophys. Res., 91, C9, 10566-10580.

Eisler, T. J., R. New and D. Calderone, 1982. Resolution and Variance in Acoustic Tomography. JASA, 72, 6, 1965-1977.

Fuglister, F. C., 1972. Cyclonic Rings formed by the Gulf Stream. Studies in Physical Oceanography. 1, ed. A. L. Gordon, Gordon and Breach Science Publishers, New York. 
Georges, T. M., R. M. Jones and J. P. Riley, 1986. Simulating Ocean Acoustic Tomography Measurements with Hamiltonian Ray Tracing. IEEE Journal of Oceanic Engineering, OE-11, 1, 58-71.

Jones, R. M., T. M. Georges and J. P. Riley, 1984. Modeling Acoustic Remote Sensing and the Florida Straits with Ray Tracing. IEEE Transactions on Geoscience and Remote Sensing, GE-22, 6, 633-640.

Jones, R. M., T. M. Georges and J. P. Riley, 1985. Parameter Estimation with Ocean Acoustic Tomography. Nature, 299, 5879, 121-125.

Jones, R. M., J. P. Riley and T. M. Georges, 1986. A Versatile Three-Dimensional Ray-Tracing Program for Acoustic Waves in an Ocean with Irregular Bottom. U. S. Department of Commerce, National Technical Information Service, Springfield, Va.

Jones, R. M., T. M. Georges and J. P. Riley, 1986. Inverting Vertical-Slice Tomography Measurements for Asymmetric Ocean Sound-Speed Profiles. Deep-Sea Research, 33, 5, 601-619.

Lai, D. Y. and P. L. Richardson, 1977. Distribution and Movement of Gulf Stream Rings. J. Phys. Oceanogr., 7, 670-683.

Liebelt, P. B., 1967. An Introduction to Optimal Estimation. Addison Wesley, Reading, Mass.

Malanotte-Rizzoli, P., J. L. Spiesberger and M. Chajes, 1985. Gulf Stream Variability for Acoustic Tomography. Deep-Sea Research, 32, 2, 237-250.

Mercer, J. A. and J. R. Booker, 1983. Long-Range Propagation of Sound Through Oceanic Mesoscale Structures. J. Geophys. Res., 88, C1, 688-699.

Mercer, J. A., W. J. Felton and J. R. Booker, 1985. Three-dimensional Eigenrays through Ocean Mesoscale Structure. JASA, 78, 1, 157-163.

Munk, W. H. and C. Wunsch, 1979. Ocean Acoustic Tomography: A Scheme for Large Scale Monitoring. Deep-Sea Research, 26A, 123-161. 
Munk, W. H., 1979. Horizontal Deflection of Acoustic Paths by Mesoscale Eddies. $J$. Phys. Oceanogr., 10, 596-604.

Munk, W. H. and C. Wunsch, 1982. Up-Down Resolution in Ocean Acoustic Tomography. Deep-Sea Research, 29, 12A, 1415-1436.

Pickard, G. L. and W. J. Emery, 1982. Descriptive Physical Oceanography. 4th ed., Pergamon Press, Oxford.

The Ocean Tomography Group, 1982. A Demonstration of Ocean Acoustic Tomography. Nature, $\mathbf{2 9 9}, 5879,121-125$.

Richardson, P. L., 1976. Gulf Stream Rings. Oceanus, 19, 3, 65-68.

Richardson, P. L., R. E. Cheney and L. V. Worthington, 1978. A Census of Gulf Stream Rings, Spring 1975. J. Geophys. Research, 83, C12, 6136-6144.

Robinson, A. R., 1983. Eddies in Marine Science. Springer-Verlag, New York.

Spiesberger, J. L., T. G. Birdsall, K. Metzger, R. A. Knox, C. W. Spofford and R. C. Spindel, 1983. Measurements of Gulf Stream Meandering and Evidence of Seasonal Thermocline Development Using Long-Range Acoustic Transmissions. $J$. Phys. Oceanogr., 13, 1836-1846.

Spiesberger, J. L. and P. F. Worcester, 1983. Perturbations in Travel Time and Ray Geometry due to Mesoscale Disturbances: A Comparison of Exact and Approximate Calculations. $J A S A, \mathbf{7 5}, 5,1443-1450$.

Spiesberger, J. L., P. Malanotte-Rizzoli and E. B. Welsh, 1985. Travel Time and Geometry of Steep Acoustic Rays Subject to Gulf Stream Variability. JASA, 78, 1, 260-263.

Spiesberger, J. L., 1985. Ocean Acoustic Tomography: Travel Time Biases. JASA, 77, 1, 83-100.

Spindel, R. C. and J. L. Spiesberger, 1981. Multipath Variability due to the Gulf Stream. $J A S A, \mathbf{6 9}, 4,982-988$. 
Spindel, R. C., 1982. Ocean Acoustic Tomography: A New Measuring Tool. OCEANUS, 25, 2, 12-21.

Urick, R. J., 1975. Principles of Underwater Sound. 2nd ed., McGraw-Iill Book Co., New York. 